\title{
活性氧响应型抗肿瘤前药研究进展
}

\author{
张留伟陈麒先* 王静云* \\ (大连理工大学生物工程学院 大连 116024)
}

\begin{abstract}
摘要 活性氧(ROS)在机体信号转导和代谢中起着至关重要的作用, 而 ROS 水平的升高与多种病变(癌症和炎症等)息 息相关, 基于肿瘤组织高水平 ROS 开发的肿瘤特异杀伤性前药策略, 在增强药效和药物选择性方面提供了一种新颖的 方法. 本综述介绍了目前用于构建抗肿瘤前药的 ROS 敏感键: 芳基喼酸/酯、烷基硫/硒醚、硫缩酮、过氧草酸酯、氨 基丙烯酸酯、噻唑烷酮和 $\alpha$-酮酰胺等，并且详叙了基于这些敏感键设计的前药在抗肿瘤方向上的应用，同时探讨了现 有 ROS 响应型前药系统的研究进展和局限性, 并对未来的研究方向进行了展望.
\end{abstract}

关键词 活性氧; ROS 响应; 前药; 药物递送; 肿瘤治疗

\section{Advances in Reactive Oxygen Species Responsive Anti-cancer Prodrugs}

\author{
Zhang, Liuwei Chen, Qixian* Wang, Jingyun* \\ (School of Bioengineering, Dalian University of Technology, Dalian 116024)
}

\begin{abstract}
Reactive oxygen species (ROS) are categorized as a class of instantaneous intermediate products of oxygen, which are usually produced by a single electron continuous reduction of $\mathrm{O}_{2}$. Examples include hydrogen peroxide $\left(\mathrm{H}_{2} \mathrm{O}_{2}\right)$, superoxide anion $\left(\mathrm{O}_{2}^{-}\right)$, hydroxyl radical $(\mathrm{HO} \bullet)$, hypochlorite radical $\left(\mathrm{OCl}^{-}\right)$and singlet oxygen $\left({ }^{1} \mathrm{O}_{2}\right)$. The endogenous ROS arise from three major resources: mitochondrial electron transport chain (Mito-ETC), endoplasmic reticulum (ER) and NADPH oxidase (NOX). The produced ROS play vital roles in physiological functions including modulation of functions of proteins, regulation of cell signaling, mediation of inflammation, and elimination of pathogens. However, the cumulative ROS level in vivo could elicit oxidative stress, which is implicated in a multitude of diseases including cancer, autoimmune diseases, inflammation, cardiovascular diseases, neurodegenerative diseases. This abnormal biochemical alteration in tumors has inspired researchers to exploit the relatively high levels of ROS for development of ROS-responsive prodrug systems. In recent years, ROS-responsive prodrug systems based on a spectrum of ROS-sensitive linkers have been designed and developed with aim of precision tumor therapy. Herein, in this review, we would like to illustrate ROS-sensitive linkers developed to date including arylboronic acid or ester, alkyl thioether or selenide, thioketal, peroxalate ester, aminoacrylate, thiazolidinone and $\alpha$-ketoamide, and elucidate the underlying molecular oxidation mechanism. Furthermore, the design of ROS-responsive prodrugs based on these sensitive linkers and their applications in anti-cancer therapy were reviewed. Additionally, the existing problems and the future research perspectives of prodrug systems were also discussed.
\end{abstract}

Keywords reactive oxygen species; ROS-responsive; prodrug; drug delivery; anti-cancer therapy

\section{1 引言}

活性氧(reactive oxygen species, ROS)是一种瞬时的 氧中间产物, 具有较高氧化活性. 常见的 ROS 包括过氧 化氢 $\left(\mathrm{H}_{2} \mathrm{O}_{2}\right)$ 、超氧阴离子 $\left(\mathrm{O}_{2}^{-}\right)$、羟基自由基 $(\mathrm{HO} \bullet)$ 、次 氯酸根 $\left(\mathrm{OCl}^{-}\right)$和单线态氧 $\left({ }^{1} \mathrm{O}_{2}\right)$ 等 $^{[1,2]}$. 内源性 $\mathrm{ROS}$ 主要 来自于线粒体电子传递链 (mitochondrial electron transport chain, Mito-ETC)、内质网(endoplasmic reticulum, ER)和 NADPH 氧化酶(NADPH-oxidase, NOX). 大 部分 ROS 的最初来源都是 $\mathrm{O}_{2}^{-}$, 并通过胞内和胞外超 氧化物歧化酶(superoxide dismutase, SOD)转化成 $\mathrm{H}_{2} \mathrm{O}_{2}$, 产生的 $\mathrm{H}_{2} \mathrm{O}_{2}$ 可以通过髓过氧化物酶(myeloperoxidase, $\mathrm{MPO}$ )和 $\mathrm{Fe}^{2+}$ 等过渡金属进一步转化为 $\mathrm{OCl}^{-}$和 $\mathrm{HO} \cdot$ (图
式 1$)^{[3]}$. 在生理状态下, ROS 能够维持在一个稳定的范 围内, 在调节细胞信号、清除细菌、介导炎症和调节蛋 白质功能等代谢途径中发挥着重要作用 ${ }^{[4-6]}$. 然而, 体内 ROS 水平的失衡可能导致氧化应激状态，从而造成不 可逆的蛋白质、脂类和 DNA 非特异性损伤 ${ }^{[7,8]}$. 氧化应 激与许多病理生理条件有关，包括癌症、自身免疫性疾 病、炎症、心血管疾病、神经系统疾病等多种疾病 ${ }^{[9-15]}$. 研究表明健康细胞的胞外 $\mathrm{H}_{2} \mathrm{O}_{2}$ 浓度在 $0.5 \sim 7 \mu \mathrm{mol} / \mathrm{L}$ 之 间，而在病理条件下的 $\mathrm{H}_{2} \mathrm{O}_{2}$ 浓度可以达到 1.0 $\mathrm{mmol} / \mathrm{L}^{[8,16-19]}$. 值得注意的是, 这种 $\mathrm{ROS}$ 水平的差异为 研究者设计前体药物提供了依据. 前药(prodrug)是指药 物经过化学结构修饰后得到的在体外无活性或活性较 小的一类药物, 在体内能够经物理、化学或酶激活后释

\footnotetext{
*E-mail: qixian@dlut.edu.cn; wangjingyun67@dlut.edu.cn; Tel.: 0411-84706805 Received April 24, 2020; published June 8, 2020.

Project supported by the National Natural Science Foundation of China (No. 21878041) and Talent Project of Revitalizing Liaoning (XLYC1807184). 项目受国家自然科学基金面上项目(No. 21878041)和兴辽英才计划基金项目(XLYC1807184)资助.
} 
放出活性药物而发挥药效 ${ }^{[20,21]}$. 众所周知, 基于抗癌药 物的化学疗法是目前最为有效的癌症治疗方法, 与手 术、放疗并驾齐驱被称为癌症的三大治疗手段. 临床中 使用的小分子抗癌药物虽然对癌细胞有较好的杀伤作 用, 但是由于其较低的溶解度、较差的稳定性以及缺乏 选择性等缺点, 使得小分子抗癌药物在体内代谢速度 快、半衰期较短、更容易被快速排出体外. 为达到抑制 和消融肿瘤的目的, 临床上多采用加大给药剂量的方式 提高肿瘤部位的药物浓度, 但是对正常组织却造成较为 严重的毒副作用. 前药主要利用癌症与正常细胞之间的 生物学差异, 在特定部位再生为活性形式, 并选择性地 恢复细胞毒性. 因此, 前药体系在药物配方和递送的各 种障碍方面提供了解决的可能性, 例如提高水溶性、改 善药代动力学、延长药物作用时间、降低药物毒副作用 以及增强药物对病灶部位的特异性等. 目前, 全球批准 上市的药物中约有 $10 \%$ 以前药的形式出现. 基于 ROS 敏感键设计的 ROS 响应型前药, 能够响应于病灶部位 高水平的 ROS, 被特异性地激活释放活性药物, 并最终 获得对癌/炎症细胞选择性杀伤、按需给药的效果(图式 2).

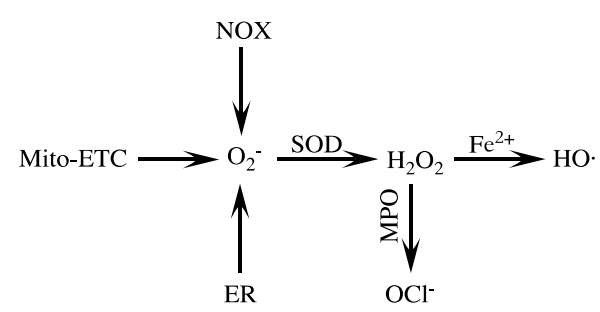

图式 1 细胞 ROS 产生的简化示意图

Scheme 1 Simplified diagram to illustrate the reaction paths of ROS in the cells

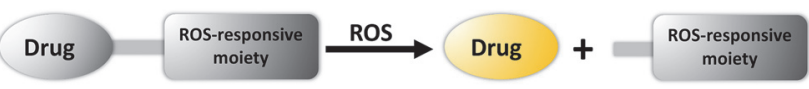

图式 2 ROS 响应性前药概念的示意图

Scheme 2 Schematic diagram to illustrate the design concept of ROS-responsive prodrugs

自 2010 年第一例 ROS 响应型前药被报道以来, ROS 响应型前药用于抗肿瘤治疗的报道层出不穷, 尤 其是近几年相关的研究论文报道数量激增, 不同的 ROS 响应机理被用于前药的设计和制备, 并取得较好 的抗肿瘤效果, 然而, ROS 响应型前药相关的文献综述 却鲜有报道. 为此, 本文综述了用于设计和制备抗肿瘤 治疗 ROS 响应型前药的敏感键及其响应机理, 并对目 前开发的 ROS 激活前药策略进行综述, 探讨现有 ROS 响应型前药系统存在的问题及对未来的该领域研究方 向进行了展望, 为进一步深入研究以及临床应用提供参 考与理论依据.

\section{ROS 响应型的敏感键}

ROS 响应型抗肿瘤前药是由抗癌药物、ROS 敏感 键及其他功能单元组合而成, 旨在掩盖药物的原有细胞 杀伤活性, 而在肿瘤部位能够响应肿瘤特异性高水平的 ROS，并触发敏感键断裂从而释放活性药物，最终达到 高效的杀伤肿瘤细胞的目的. 目前用于设计 ROS 响应 型前药的敏感键主要有(图式 3)：芳基硼酸/酯 (arylboronic acid or ester)、烷基硫/硒醚(alkyl thioether or selenide)、硫缩酮(thioketal, TK)、过氧草酸酯(peroxalate ester)、氨基丙烯酸酯(aminoacrylate, AA)、噻唑烷酮 (thiazolidinone, TZ)和 $\alpha$-酮酰胺 ( $\alpha$-ketoamide)等. ROS 敏 感键的氧化断裂释药的机理如图式 3 所示, 由芳基硼酸/ 酯(包括苯硼酸、苯硼酸酯和香豆素硼酸酯等)形成的前 药, 通过 ROS 和硣原子配位, 氧化 $\mathrm{B}-\mathrm{C}$ 键形成硼酸盐 和芳基酚, 芳基酚进一步自断裂形成醌类物质释放出分 子药物; 由烷基硫/硒醚连接的前药，疏水的二价硫/硒 容易被 ROS 氧化为亲水的四价或六价的硫/硒，含有硫/ 硒醚的前药在相变过程中容易被水解释放分子药物; 由 硫缩酮偶联形成的前药，在 ROS 环境中，硫缩酮键易被 氧化而断裂, 生产的颈基发生自断裂释放出分子药物; 由过氧草酸酯形成的前药, 氧草酸酯键易被 ROS 氧化 生成 1,2-二氧乙二酮并迅速断裂分解为二氧化碳并释放 分子药物; 由氨基丙烯酸酯形成的前药, 其中富电子烯 烃易被 ROS 氧化，进行[2+2]环加成反应，进而自断裂 释放分子药物; 由噻唑烷酮或 $\alpha$-酮酰胺基团掩蔽羧基形 成的前药，噻唑烷酮/ $\alpha$-酮酰胺易被 ROS 氧化攻击, 酰 胺键水解促使屏蔽基团离去释放分子药物. 在用于

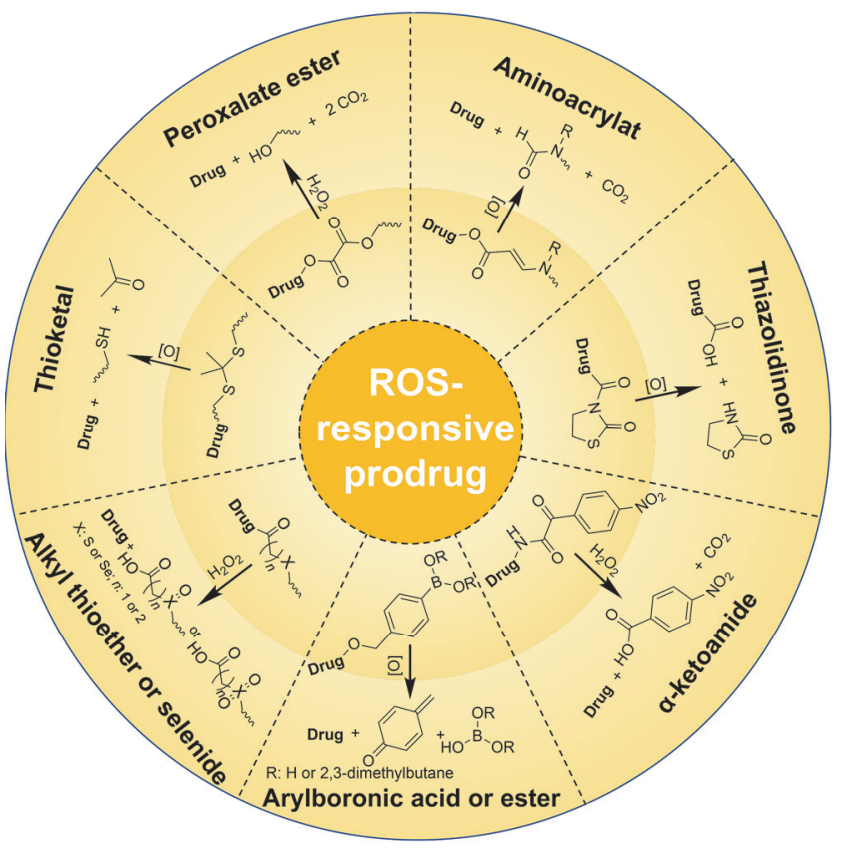

图式 3 ROS 敏感的前药连接键及其氧化机制

Scheme 3 ROS-sensitive linkers in fabrication of prodrugs and the oxidation mechanisms 
ROS 响应型前药设计的敏感键中, 芳基硼酸/酯、噻唑烷 酮和 $\alpha$-酮酰胺基团由于其为端位基团, 多用于直接与药 物分子的活性基团(氨基、羟基和羧基等)连接, 以屏蔽 药物分子的原有活性; 此外, 烷基硫/硒醚、硫缩酮、过 氧草酸酯和氨基丙烯酸酯基团易作为连接臂, 在两端共 价连接不同特性的材料, 能够设计出多功能的前药, 在 ROS 响应型前药的应用显得更为灵活. 研究者们根据 ROS 敏感键的响应机理, 选择合适的药物分子和载体 材料, 设计和制备了用于抗肿瘤治疗的不同类型前药, 并评估了 ROS 响应型前药的体外/体内抗肿瘤效果, 本 文将对其进行介绍和总结便于对该领域有更好的理解.

\section{ROS 响应型抗肿瘤前药}

\section{1 芳基硼酸/酯基前药}

芳基硼酸/酯能够与 $\mathrm{H}_{2} \mathrm{O}_{2}$ 等活性氧发生反应, 主要 通过 $\mathrm{H}_{2} \mathrm{O}_{2}$ 与硼原子配位, 氧化 $\mathrm{B}-\mathrm{C}$ 键形成硼酸盐, 在 水中可迅速水解为嗍酸/酯和芳香酚(可转化为醌类物 质), 同时释放出活性分子(图式 3), 该机制被广泛应用 于苂光探针(fluorescent probe) ${ }^{[22,23]}$ 和前药的设计及制备. 本节介绍芳基硼酸及其衍生物在 ROS 激活前药策略中 的应用.

Cohen 等 ${ }^{[24]}$ 在利用芳基嗍酸酯作为 ROS 响应型前 药方面做了开拓性的探索工作, 2010 年, Cohen 等首次 将芳基硼酸酯与两种基质金属蛋白抑制剂(matrix metalloproteinase inhibitor, MMPi)偶联得到两种前药(图 1a), 用于脑卒中后缺血再灌注损伤的保护性治疗, 在氧化条 件下 $\left(100 \mu \mathrm{mol} / \mathrm{L} \mathrm{H}_{2} \mathrm{O}_{2}\right)$ 获得了与原药一致的抑制效果. 随着第一例 ROS 响应型前药的报道, 引起了同行业研 究者的密切关注, ROS 响应型抗肿瘤前药的设计及其抗 肿瘤性能研究的报道不断涌现. 从 2011 年开始, Peng 课 题组 ${ }^{[25-31]}$ 在 ROS 激活抗癌药物氮芥类前药方面做了很 多研究, 并通过芳基硼酸/酯与氮芥或芳香氮芥共价连 接形成一系列氮芥前药用于抗肿瘤的研究. 例如, 在 2018 年, Peng 等 ${ }^{[26]}$ 通过苯硼酸与氮芥共价链接, 并在苯 环上引入半胱氨酸甲酯(methyl cysteinate)增加其水溶性 和渗透性形成了 ROS 激活的氮芥前药 10(图 1b), 体外 对乳腺癌细胞系(MDA-MB-468)和白血病细胞系(CLL) 细胞毒性结果表明, 前药 10 具有较低的 $\mathrm{IC}_{50}$ 值(3.43 $\mu \mathrm{mol} / \mathrm{L})$, 其毒性是临床化疗药物苯丁酸氮芥 (chlorambucil, $\mathrm{CHL}, \mathrm{IC}_{50}=48.7 \mu \mathrm{mol} / \mathrm{L}$ ) 和米尔法兰 (melphalan, $\mathrm{IC}_{50}=34.44 \mu \mathrm{mol} / \mathrm{L}$ ) 毒性的 $10 \sim 14$ 倍，同时 氨基酸侧链的引入提高了前药 10 的溶解度和组织渗透 性; 体内结果显示前药 10 能够显著抑制小鼠 MDA-MB-468 实体瘤的生长, 而且没有明显的系统毒 性, 实现了 ROS 激活的氮芥前药体内的肿瘤治疗. 在 ROS 响应型氨基二茂铁(aminoferrocene, AF) 前药的设计 和应用方面, Mokhir 等 ${ }^{[32-37]}$ 报道了很多相关的工作, 将 ROS 敏感的苯嗍酸酯与 AF 结合制备了一系列 AF 前药
系统，在氧化条件下， $\mathrm{AF}$ 转化为氨基二茂铁离子，氨基 二茂铁离子分解释放出 $\mathrm{Fe}^{2+}$ 引起芬顿反应(Fenton reaction) ${ }^{[38]}$, 可以诱导氧化应激并最终导致细胞死亡(图 2a).
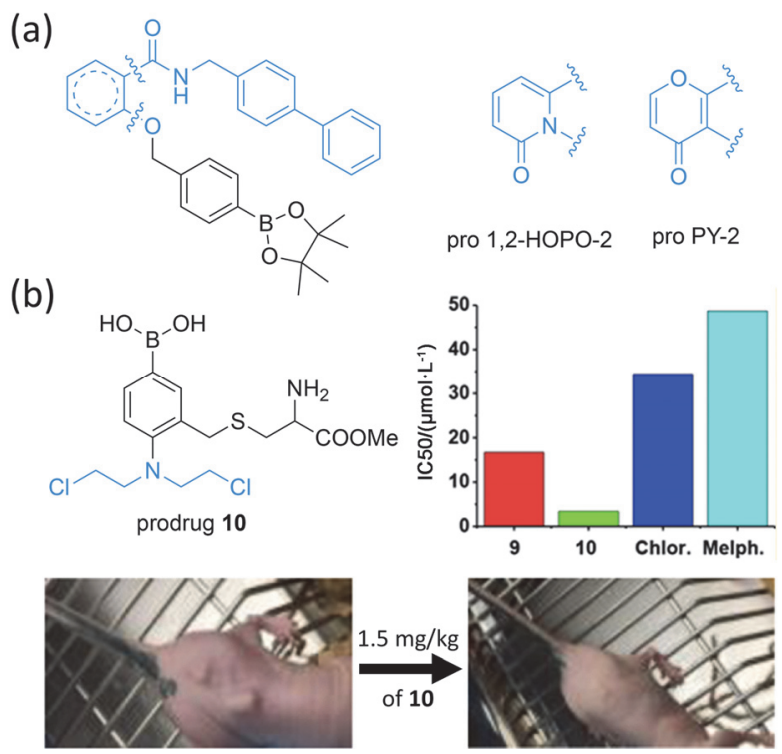

图 1 (a) 两种 ROS 激活苯嗍酸酯基的基质金属蛋白抑制剂前药化学 结构 ${ }^{[24]}$; (b) ROS 响应的苯嗍酸基氮芥前药 10 及其对 MDA-MB-468 细胞的 $\mathrm{IC}_{50}$ 和荷瘤小鼠治疗效果图 ${ }^{[26]}$.

Figure 1 (a) Chemical structures of two ROS-activated matrix metalloproteinase inhibitor prodrugs ${ }^{[24]}$; (b) ROS-responsive nitrogen mustard (prodrug 10), $\mathrm{IC}_{50}$ of $\mathbf{1 0}$ against MDA-MB-468 cells and therapeutic outcomes in treatment of tumor-bearing mice ${ }^{[26]}$. Copyright (2018) American Chemical Society.

(a)
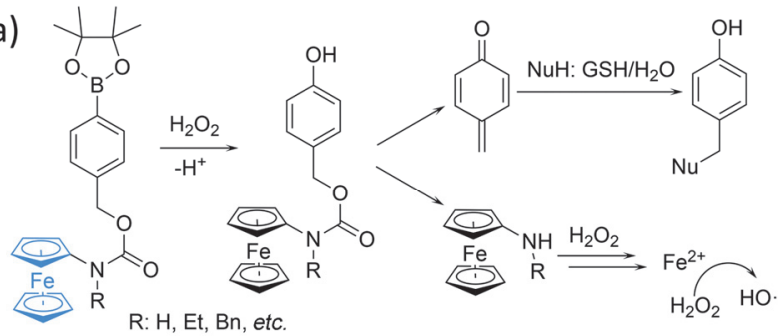

(b)
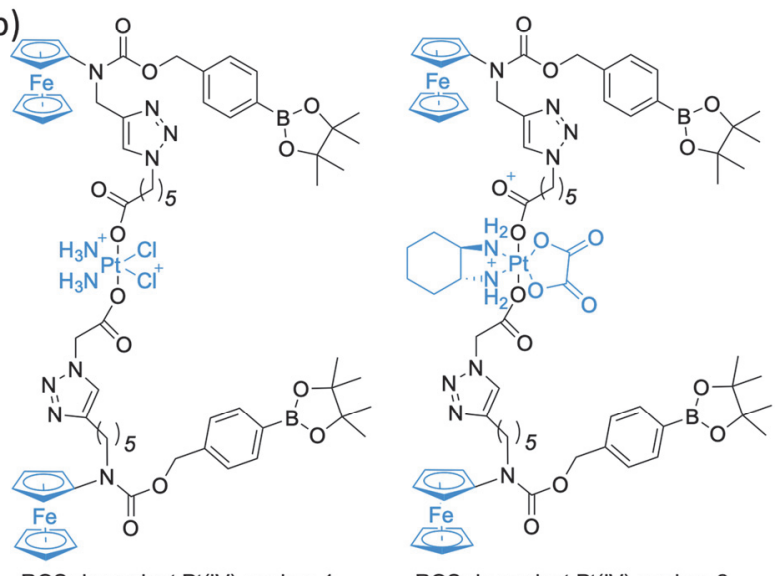

ROS-dependent Pt(IV) prodrug 1

图 2 (a) $\mathrm{AF}$ 前药的化学结构及其被 $\mathrm{H}_{2} \mathrm{O}_{2}$ 氧化机理 ${ }^{[32-37]}$; (b) $\mathrm{ROS}$ 响应 型三组分 $\mathrm{Pt}(\mathrm{IV})$ 前药结构式 ${ }^{[39-41]}$.

Figure 2 (a) Chemical structure of aminoferrocene prodrug and its activation in presence of hydrogen peroxide ${ }^{[32-37]}$; (b) Chemical structure of ROS-dependent three-component system Pt(IV) prodrug $\mathbf{1}$ and $\mathbf{2}^{[39-41]}$. 
在 2017 2018 年, 该课题组 ${ }^{[39-41]}$ 设计了更为复杂的 $\mathrm{AF}$ 前药系统, 将芳基硼酸酯修饰的 AF 前药与 Pt(IV)配合 物偶联形成三组分 (AF-Pt-AF) 前药系统(图 2b), 经活化 后分别释放铂类药物和铁离子实现协同治疗效果; 实验 结果证明了前药对卵巢癌细胞株(A2780)和顺铂耐药细 胞株(A2780cis)都有显著毒性, 但是对人成纤维细胞 $(\mathrm{HDFa})$ 几乎无细胞毒性, 从而证明了前药具有较低的 系统毒性.

对现有的抗癌药物进行修饰和改造开发新的 ROS 激活的前药系统的报道层出不穷, 可以赋予原抗癌药物 新的特性: 屏蔽或降低正常细胞毒性、更好的溶解性、

较长的血液循环时间、近红外成像和肿瘤细胞靶向性 等 $^{[42-53]}$. 早在 2014 年, Hong 等 ${ }^{[46]}$ 就利用硽酸酯基香豆 素荧光团遮蔽抗癌药物 7-乙基-10-羟基喜树碱 (7-ethyl-10-hydroxycamptothecin, SN-38) 的活性, 制备 $\mathrm{H}_{2} \mathrm{O}_{2}$ 激活的多功能前药 pro SN38(图 3), 实验结果证明 了该前药在小鼠黑色素瘤(B16F10)和 HeLa 细胞中被 $\mathrm{H}_{2} \mathrm{O}_{2}$ 激活显示出与 $\mathrm{SN}-38$ 相近的细胞毒性(无 $\mathrm{H}_{2} \mathrm{O}_{2}$ 时, pro SN38 对细胞存活率提高了 2 倍), 同时释放的香豆 素能够在癌细胞中苂光成像, 在小鼠转移性肺肿瘤模型 (B16F10)中表现出高效的抗肿瘤活性. 随后, $\mathrm{Lu}$ 等 ${ }^{[47]}$ 在 2016 年将硼酸直接取代 SN-38 的 10 位羟基获得前药, 在体外乳腺癌(MCF-7)细胞和体内癌症模型(U87MG)中 获得类似的效果. Zhang 等 ${ }^{[48]}$ 也在 2017 年通过用苯硼酸 酯基共价连接近红外光敏剂(NIR photosensitizer, NPS) 和抗癌药物 5'-脱氧-5-氟尿苷(5'-deoxy-5-fluorouridine, 5'-DFUR) 形成无荧光和细胞毒性的多功能前药 pro 5'-DFUR(图 3), 在 $\mathrm{H}_{2} \mathrm{O}_{2}$ 存在下嗍酸酯基团被破坏, 释 放出荧光和光动力性能恢复的 NPS 以及活性抗癌药物 5'-DFUR, 实验结果表明, 其对癌细胞(HeLa, HepG2)和 荷瘤裸鼠具有优于原药的肿瘤抑制效果, 结合光敏剂的 光动力治疗和近红外成像等功能实现联合治疗肿瘤的
目的. 在 2018 年, Tang 等 ${ }^{[49]}$ 报道了将苂光探针羧化四苯 基乙烯 (tetraphenylethylene, TPE)和抗癌药物阿霉素 (doxorubicin, DOX)通过硼酸酯共价连接形成一种三组 分的多功能前药 pro DOX(图 3), 并观察到在 $\mathrm{H}_{2} \mathrm{O}_{2}$ 存在 时前药在细胞中的苂光成像, 以及对 HeLa 细胞杀伤的 增强效果. 在此之后, Obika 等 ${ }^{[50]}$ 在 2019 年设计合成了 一种 ROS 激活的吉西他滨(gemcitabine, GEM)前药 pro $\operatorname{GEM}$ (图 3), 人胰腺癌细胞(PSN1)中高浓度的 $\mathrm{H}_{2} \mathrm{O}_{2}$ 能够 特异性地氧化苯硣酸酯释放 GEM 并显示出显著的癌细 胞毒性(有 $\mathrm{H}_{2} \mathrm{O}_{2}$ 存在时, 细胞存活率与原药接近), 在荷 瘤裸鼠的肿瘤治疗中显示出与 GEM 等同的抑制效果和 更低毒副作用. 2018 2020 年期间, Clausen 等 ${ }^{[51,52]}$ 将氨 基喋呤(aminopterinum, AMT)和甲氨蝶呤(methotrexate, MTX)与芳基嗍酸偶联, 分别形成对应的前药 pro AMT 和 pro MTX(图 3), 结果显示前药对 MCF-7 和肺癌 (NCIH-460)细胞系有 ROS 激活的细胞毒性, 并且与原 药相比, 前药具有更高的安全性. Zhang 等 ${ }^{[53]}$ 在 2020 年 初报道了一个对现有抗癌药物藤黄酸 (gambogic acid, GA)修饰的前药分子, 将 GA 与苯硼酸酯偶联形成 ROS 激活的前药 pro GA(图 3), 该前药体系能够在膀胱癌细 胞(MB49)中响应高水平 ROS 快速释药, 表现出与 GA 类似的细胞毒性 $\left(\mathrm{GA}: \mathrm{IC}_{50}=0.52 \mu \mathrm{mol} / \mathrm{L}\right.$, pro $\mathrm{GA}: \mathrm{IC}_{50}=$ $0.99 \mu \mathrm{mol} / \mathrm{L})$, 但对正常细胞 NIH-3T3 几乎无细胞毒性; 在原位浅表性膀胱癌模型中可以显著抑制膀胱癌细胞 的增殖, 而对机体器官组织细胞没有造成损害.

药物递送系统(drug delivery system, DDS)在提高药 物的靶向性和缓释性、延长药物体内循环时间和降低药 物系统毒副作用等方面有着很好的效果, 将抗癌药物通 过与 ROS 敏感键连接进而装配成 ROS 响应型的纳米前 药在按需给药和肿瘤精准治疗方面有着明显的优 势 ${ }^{[54-61]}$. 2018 年, Jiang 等 ${ }^{[58]}$ 将 CHL 与亲水性聚乙二醇 (polyethylene glycol, PEG)和 4-(羟甲基)苯基硼酸结合
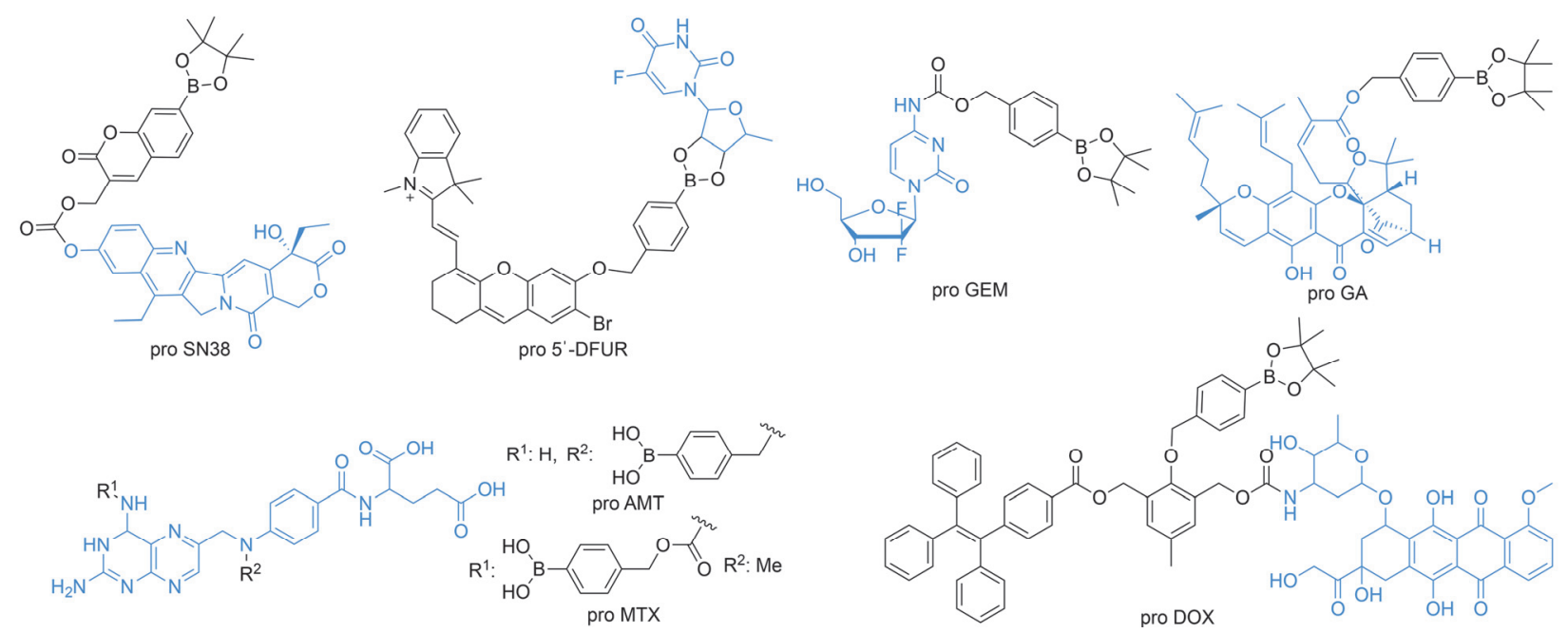

图 3 芳基硼酸/酯基 ROS 响应型前药的化学结构 ${ }^{[46-53]}$

Figure 3 Chemical structure of arylboronic acid/ester ROS-responsive prodrugs ${ }^{[46-53]}$

SN38: 7-ethyl-10-hydroxycamptothecin, 5'-DFUR: 5'-deoxy-5-fluorouridine, GEM: gemcitabine, GA: gambogic acid, AMT: aminopterin, MTX: methotrexate, DOX: doxorubicin 
制备了具有 ROS 响应的 CHL 纳米前药(图 4), 在 $\mathrm{H}_{2} \mathrm{O}_{2}$ 存在下, 纳米前药能快速释放天然 $\mathrm{CHL}$, 对 $\mathrm{MCF}-7$ 细 胞抗增殖和调亡的研究表明纳米前药胶束比 CHL 具有 更好的治疗效率(细胞调亡率分别为 $43.3 \%$ 和 $23.5 \%$ ), 在体内抑瘤效果和生物相容性方面, 该纳米前药均显示 出更好的优越性. 2020 年, Liu 等 ${ }^{[59]}$ 报道了一种基于苯硓 酸酯连接的紫杉醇(paclitaxel, PTX)和 PEG 两亲性聚合 物经自组装形成的前药胶束(图 5), 该胶束在正常生理 环境下具有很高的胶束稳定性, 但在肿瘤高水平 ROS 环境中, 胶束组装结构迅速解离和释放活性 PTX 药物; 与 PTX 相比, 该胶束在体外对人脑胶质瘤(U251)和 $\mathrm{MCF}-7$ 细胞的 $\mathrm{IC}_{50}$ 值分别降低 11 倍和 14 倍, 该前药胶 束对体内 MCF-7 肿瘤模型显示出更高的抗肿瘤活性(前 药肿瘤抑制率(tumor inhibition rate, TIR) $=89.4 \%$, PTX 的 $\mathrm{TIR}=68.5 \%$ ) 和更低的毒副作用. 同年, You 等 ${ }^{[60]}$ 合成 了一种线粒体靶向的光响应聚合物并通过疏水作用包 裹疏水的光响应型一氧化碳(CO)前药二苯基环丙烯酮 (diphenylcyclopropenone)和 AF 前药形成纳米颗粒(图 6), 在紫外光照射下可快速释放 $\mathrm{CO}$ 和 $\mathrm{AF}$ 前药, 后者能够 响应肿瘤高水平 $\mathrm{ROS}$ 快速分解为 $\mathrm{Fe}^{2+}$ 与 $\mathrm{CO}$ 协同作用 促使细胞产生更多的 ROS, 致使线粒体崩溃, 对 HeLa 和 4T1 细胞作用结果显示该体系具有肿瘤靶向递送和 协同治疗的效果. 此外, $\mathrm{Xu}$ 等 ${ }^{[61]}$ 首次报道了 $\mathrm{ROS}$ 敏感 的芳基硼酸酯通过氨基甲酸键与核糖核酸酶

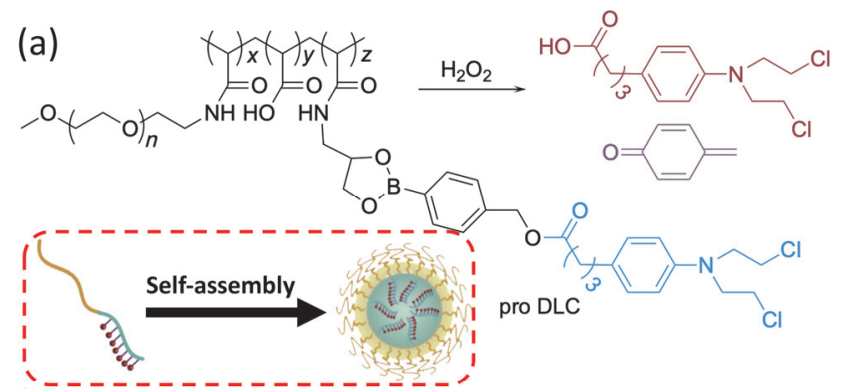

(b)
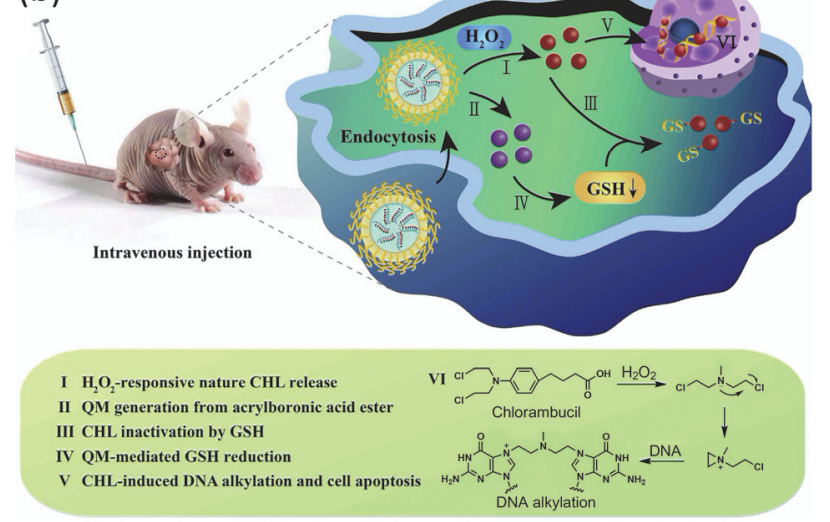

图 4 (a) ROS 响应型苯丁酸氮芥前药的化学结构及其自组装形成前 药纳米胶束, (b) 细胞内 $\mathrm{H}_{2} \mathrm{O}_{2}$ 触发药释和生成 $\mathrm{QM}$ 的示意图 ${ }^{[58]}$.

Figure 4 (a) Chemical structure of the ROS-responsive CHL prodrug and its self-assembly into prodrug micelles, (b) schematic illustration of intracellular $\mathrm{H}_{2} \mathrm{O}_{2}$-triggered drug release and quinone methide (QM) generation $^{[58]}$. Copyright (2018) Elsevier. $\mathrm{a}$ (RNase a)中的赖氨酸残基相连形成 RNase 前药，由于 遮盖了其生物活性所必需的赖氨酸残基而失去 RNase 活性, 在 ROS 高水平的 B16F10 细胞中, 苯硼酸酯氧化 水解后使其酶活重新恢复, 并显示出较高的细胞毒性.
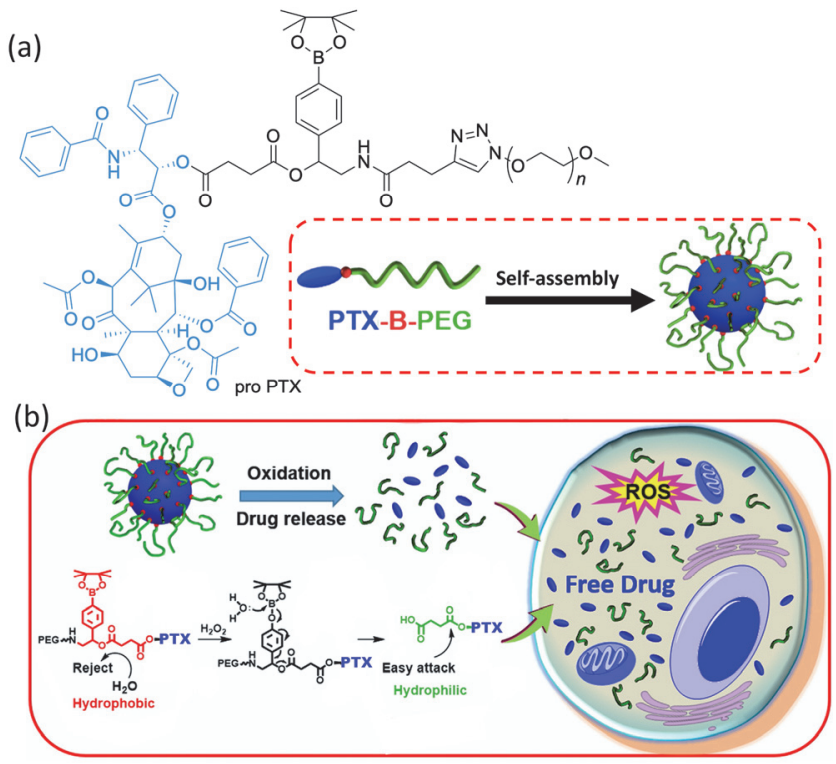

图 5 (a) ROS 响应型紫杉醇前药的化学结构及其自组装形成前药纳 米胶束, (b) 前药纳米胶束响应肿瘤 ROS 的药释机理示意图 ${ }^{[59]}$.

Figure 5 (a) Chemical structure of ROS-responsive PTX prodrug and its self-assembly into prodrug micelles, (b) prodrug-based micelle was activated by tumoral ROS to release PTX drug ${ }^{[59]}$. Copyright (2020) Elsevier.

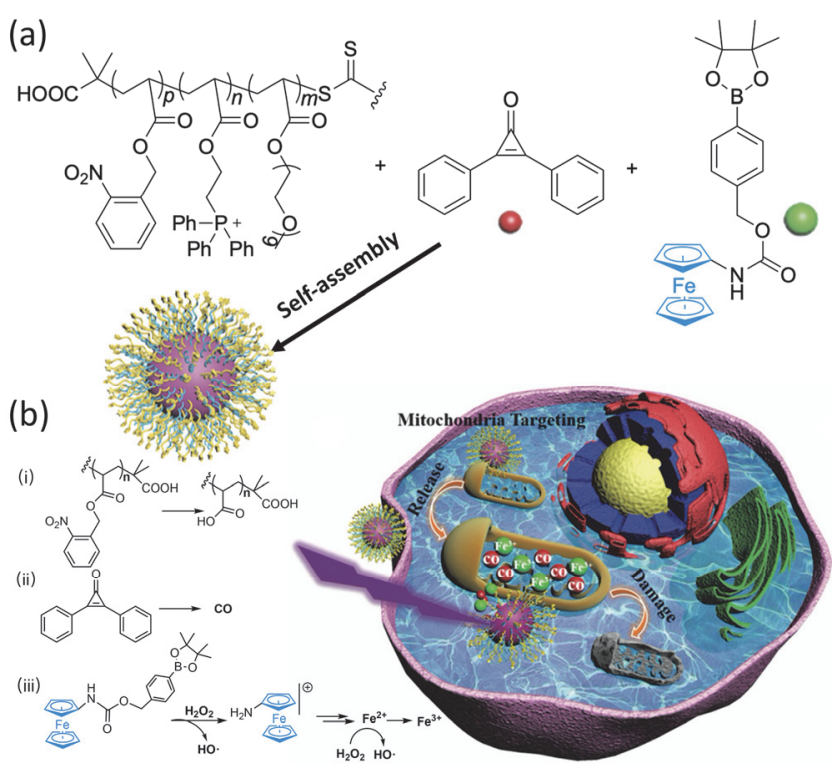

图 6 (a) ROS 响应型氨基二茂铁前药、CO 前药和两亲性嵌段共聚物 的化学结构, (b) 线粒体靶向光响应纳米颗粒的制备及其对癌细胞的 联合治疗 ${ }^{[60]}$

Figure 6 (a) Chemical structure of ROS-responsive aminoferrocene prodrug, $\mathrm{CO}$ prodrug and amphiphilic block copolymer, (b) the preparation of mitochondria-targeted photo-responsive nanoparticles and its cooperative cancer therapy ${ }^{[60]}$. Copyright $(2020)$ The Royal Society of Chemistry. 


\section{2 烷基硫/硒醚基前药}

在氧化环境中, 含硫醚的化合物中疏水的硫可以被 氧化为亲水的亚砜( sulfoxide)并最终氧化为砜( sulfone), 这种从疏水状态到亲水状态的转变促进了临近酯键的 水解, 从而释放共价连接的活性分子(图式 3). 与含硫醚 化合物类似, 含硒 $(\mathrm{Se})$ 的化合物也能够经历从二价态氧 化为四价/六价态的过程发生亲疏水性改变, 因此该机 制被开发用于 ROS 激活型前药的设计和制备.

不同于芳基硼酸/酯基前药主要以分子药物的形式 出现, 烷基硫/硒醚基前药基于其作为连接臂的特性常 被用于设计为前药纳米系统用于 ROS 触发式的抗肿瘤 治疗 ${ }^{[62-70]}$. Sun 课题组 ${ }^{[63-69]}$ 在烷基硫/硒梄基的前药方面 做了很多报道, 2017 年, Sun 等 ${ }^{[63]}$ 将 PTX 和油酸(oleic acid, OA) 疏水性小分子通过硫醚键连接的前药分子 PTX-s-OA(图 7a), 和生育酚聚乙二醇琥珀酸酯( TPGS $_{2 \mathrm{k}}$ ) 一起自组装形成前药纳米系统, 实验发现该体系不仅能 够快速响应 ROS, 也能够与 GSH 反应释放 PTX, 成为 氧化还原双响应的纳米前药系统(图 7c), 在体内人表皮 样癌异种移植瘤(KB-3-1)中具有很强的抗肿瘤活性. 将 光敏剂引入到 ROS 响应型前药系统中, 在激光照射下 增加局部的 ROS 水平, 这种有光敏剂产生的外源性 ROS 可以促进药物在肿瘤部位的快速释放和累积, 结 合光敏剂的光动力治疗 (photodynamic therapy, PDT)实 现高效地协同抗肿瘤效果. 因此, 2019 年, 该课题组 ${ }^{[64]}$ 将 PTX-s-OA 前药分子与光敏剂焦脱镁叶绿酸 a(pyropheophorbide a, PPa)PEG 偶联物 $\left(\mathrm{PPa}-\mathrm{PEG}_{2 \mathrm{k}}\right.$, 图 $7 b)$ 形成光动力和 $\mathrm{ROS}$ 响应型纳米前药组装体, 在激光 $\left(660 \mathrm{~nm}, 58 \mathrm{~mW} / \mathrm{cm}^{2}\right)$ 照射下, PPa-PEG $2 \mathrm{k}$ 产生的 ROS 不 仅可以用于 PDT, 同时能够与肿瘤细胞内高水平的 ROS 协同促进 PTX 的释放, 实验结果显示在激光照射或 $\mathrm{H}_{2} \mathrm{O}_{2}(2 \mathrm{mmol} / \mathrm{L})$ 存在下, PTX 能够快速释放, 人表皮样 癌 $(\mathrm{KB})$ 异种移植瘤小鼠的治疗中确认了光动力和化药 协同治疗效果. 同年, 为了实现高效同步共给药和协同 治疗, 该课题组 ${ }^{[65]}$ 将 PTX 和光敏剂 PPa 通过硫醚键偶联 为 ROS 响应型二聚体前药分子 PTX-s-PPa(图 7a), “二 合一” 的二聚体在水中自组装为自促进 ROS 响应型药 物释放的纳米粒子, 实验结果显示在肿瘤细胞 $(\mathrm{KB}$, A549, 4T1) 内过量产生的 ROS 与激光照射下 PPa 产生的 ROS 协同促进了 PTX 药物的按需释放, 体内荷瘤小鼠 $(\mathrm{KB}, 4 \mathrm{~T} 1)$ 治疗实验结果表明 PTX 联合 PPa 介导的 PDT 具有协同抗肿瘤作用. 光敏剂在产生 ROS 时需要激光 作为外源刺激, 在一定程度上限制了其在临床中的应 用. 同年, 为了克服光敏剂的不足, 该课题组 ${ }^{[66]}$ 将亚油 酸(linoleic acid, LA)代替 OA 合成 PTX-s-LA 前药分子 (图 7a), 与 $\beta$-拉帕醌 $(\beta$-lapachone, $\beta$-lapa)和聚乙二醇-聚 乳酸(PEG-PDLLA)共同组装为纳米颗粒, $\beta$-lapa 利用癌 细胞内特异性高表达醌氧化还原酶-1(NAD(P)H:quinone oxidoreductase I, NQO1)催化 $\mathrm{O}_{2}$ 产生 $\mathrm{ROS}$ (图 7b) 与肿
瘤细胞内源性高 $\mathrm{ROS}$ 水平结合，可以协同促进 PTX-s-LA 释放活性药物 PTX, 后期实验结果验证了该 前药纳米系统具有前药自增强的生物活性、选择性释 放、癌细胞细胞毒性、延长循环时间、增强肿瘤积聚等 特点，在荷瘤小鼠治疗中该体系能够显著抑制 4T1 肿瘤 的生长. 此外, $\mathrm{Xu}$ 等 ${ }^{\left[{ }^{[8]}\right.}$ 以马来酰亚胺(maleimide, MAL) 和 PTX 为原料, 以硫醚键为连接臂合成一种新的 ROS 敏感的 PTX 前药 PTX-s-MAL1 (图 8a), 该前药在体内通 过马来酰亚胺与白蛋白迅速结合形成前药载体, 由于肿 瘤组织处增强的渗透和保留(enhanced permeability and retention effect, EPR)效应，使得前药载体能够在肿瘤部 位累积并快速响应肿瘤微环境中 ROS 释放 PTX 药物, 小鼠乳腺癌异种移植模型结果显示肿瘤抑制率比游离 PTX 组提高了 2 倍. 随后, 在 2019 年, Sun 等 ${ }^{[69]}$ 合成了 类似的 PTX 前药 PTX-s-MAL2(图 8a), 考虑到前药分子 在血液中化学稳定性较差, 在体外将自组装的纳米前药 外包覆聚多巴胺(polydopamine)和 PEG 外衣赋予纳米

(a)

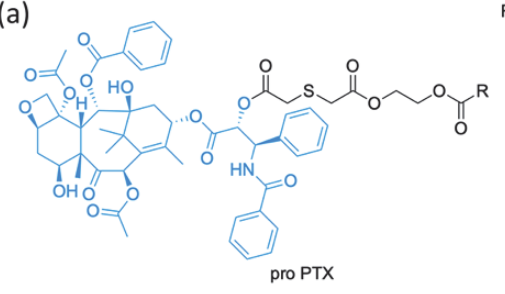

R: OA s $^{3}+\frac{7}{7}=X+\frac{1}{7}$
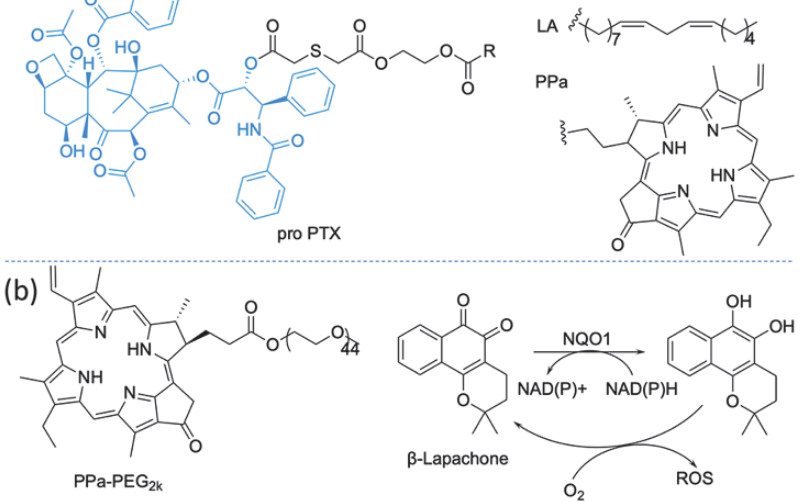

(c)
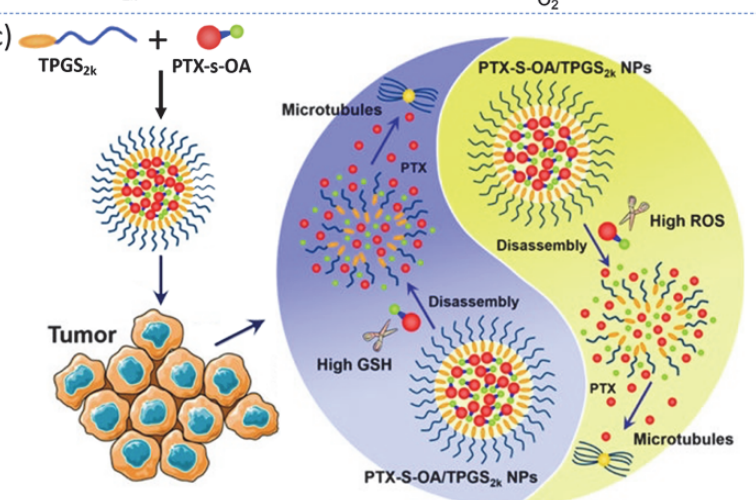

图 7 (a) ROS 响应型紫杉醇前药的化学结构 ${ }^{[63-66]}$, (b) 光敏剂 PPa 和 $\beta$-拉帕醌的化学结构及 $\beta$-拉帕醌在 NQO1 酶催化下产生 ROS 机

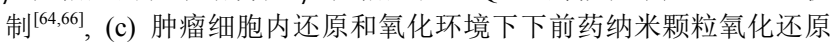
双响应药释的示意图 ${ }^{[63]}$.

Figure 7 (a) Chemical structure of ROS-responsive PTX prodrugs PTX-s-OA, PTX-s-LA and PTX-s-PPa ${ }^{[63-66]}$, (b) chemical structure of photosensitizers $\mathrm{PPa}$ and $\beta$-lapachone, and ROS generation by $\beta$-lapachone under the catalysis of NQO1 enzyme ${ }^{[64,66]}$, (c) schematic representation of redox dual-responsive drug release of prodrug PTX-s-OA in presence of two opposite stimuli within tumor cells ${ }^{[63]}$. Copyright (2016) American Chemical Society.

OA: oleic acid, LA: linoleic acid, PPa: pyropheophorbide a 

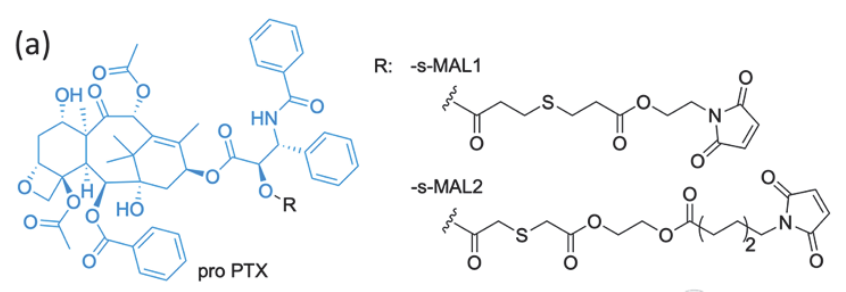

(b)

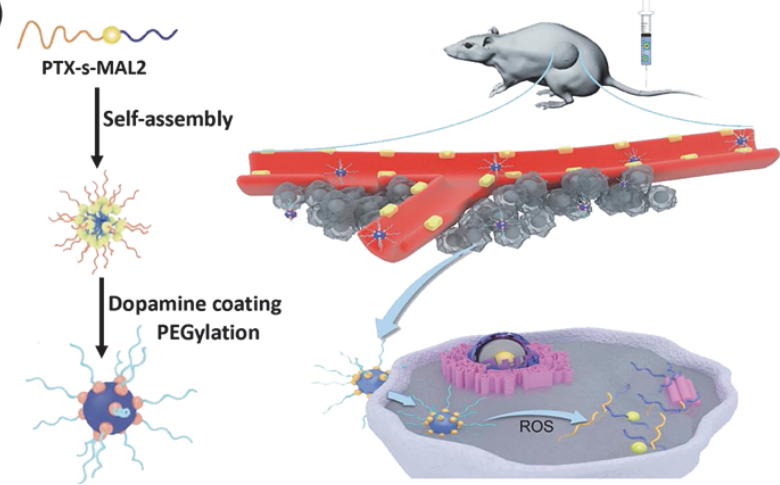

图 8 (a) ROS 响应型紫杉醇前药的化学结构 ${ }^{[6,69]}$, (b) 前药纳米粒自 组装与肿瘤细胞化疗示意图 ${ }^{[69]}$.

Figure 8 Chemical structure of the ROS-responsive PTX prodrugs PTX-s-MAL1 and PTX-s-MAL2 ${ }^{[68,69]}$, (b) schematic illustration of self-assembly of prodrug nanoparticles and the chemotherapy of tumor cells $^{[69]}$. Copyright (2019) The Royal Society of Chemistry.

MAL: maleimide

前药高溶解性和稳定性(图 8b), 体外实验验证了该体系 对 $\mathrm{H}_{2} \mathrm{O}_{2}$ 较高的敏感性, 能够在肿瘤细胞中高水平 ROS 环境选择性地快速释放 PTX, 但对正常细胞的毒性较 小，在体内实验中，该系统明显增强了前药在肿瘤部位 富集, 并显示出对 4T1 肿瘤模型显著的治疗效果和对机 体较低的毒副作用.

研究发现硫醚键偶联的前药分子不仅能够在 ROS 环境下通过氧化 S 原子为亲水性(亚)砜促进酯键水解释 放药物分子, 也可以在还原环境下通过 GSH 等物质的 颈基攻击酯键直接硫解出药物分子(图 9a), 显示出肿瘤 微环境双响应快速释药的潜力 ${ }^{[63,71-73]} .2013$ 年, Shen 等 ${ }^{[71]}$ 用硫醚键偶联抗癌药物 SN-38 和低聚乙二醇(oligo ethylene glycol, OEG)形成前药 SN38-s-OEG(图 9b)进而 自组装为纳米颗粒, 实验结果证明了前药系统能够对 $\mathrm{H}_{2} \mathrm{O}_{2}, \mathrm{GSH}$ 和光敏剂(Ce6)产生的 ROS 具有响应性释药 行为, 体内乳腺癌(Bcap37)移植瘤小鼠治疗结果表明该 体系具有显著的抑瘤和抗转移能力, 从而有效地延长荷 瘤小鼠的存活率和存活时间. 2019 年, Kim 等 ${ }^{[72]}$ 构建了 依赖于两种肿瘤微环境因子(GSH 和 $\mathrm{H}_{2} \mathrm{O}_{2}$ ) 激活的前药, 通过硫醚键将 SN-38 和环氧合酶-2(cyclooxygenase-2, COX-2)抑制剂吲哚美辛 (indomethacin, IDM) 偶联形成 分子前药 SN38-s-IDM(图 9b), 在 COX-2 阳性的侵袭性 结肠癌细胞(SW620 和 LoVo)中观察到细胞对前药较高 的摄取能力, 相比于单独使用 SN-38 和 SN-38 与 IDM 联用实验组, 前药分子的抗癌活性分别提高了 5 倍和 3 倍, 在异种移植瘤(SW620)模型上, 前药分子展示出显
著的抑瘤效果和无明显的系统毒性. 2020 年, $\mathrm{Ge}$ 等 ${ }^{[73]}$ 通 过硫醚键在聚乙二醇-聚甲基丙烯酸甲酯侧链上连接喜 树碱(camptothecin, CPT)组成的 GSH 和 ROS 双响应两 亲性嵌段共聚物前药 CPT-s-PEG(图 9b), 并自组装成纳 米颗粒 (图 9c), 该前药体系对 HeLa 细胞有较低的 $\mathrm{IC}_{50}\left(6.3 \mu \mathrm{mol} / \mathrm{L}, \mathrm{GSH}\right.$ 和 $\mathrm{ROS}$ 单一刺激的前药的 $\mathrm{IC}_{50}$ 分 别为 17.8 和 $28.9 \mu \mathrm{mol} / \mathrm{L}$ ), 在体内抗 $\mathrm{H} 22$ 肿瘤方面, 前 药拥有较长的血液循环和更多的肿瘤部位富集, 并表现 出更强的肿瘤抑制作用.

(a)
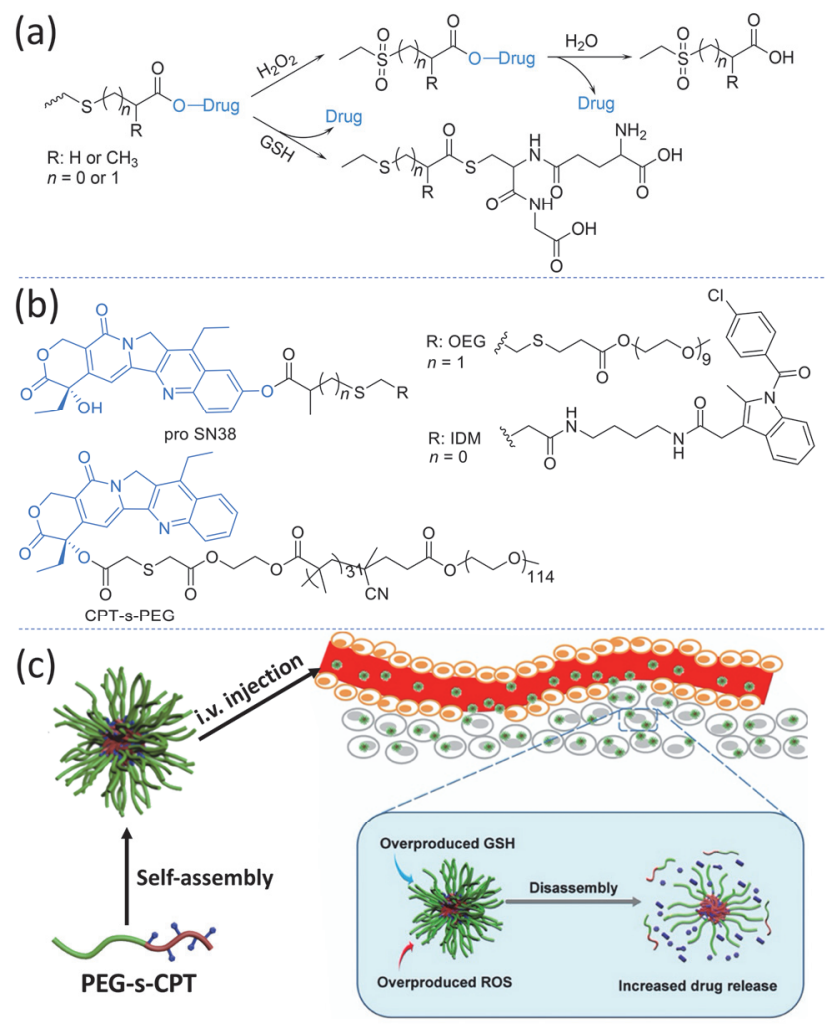

图 9 (a) 前药分子通过 GSH 硫解和 ROS 氧化的药物释放机制 ${ }^{[71-73]}$, (b) ROS 响应型喜树碱前药的化学结构 ${ }^{[71-73]}$, (c) CPT-s-PEG 前药分子 自组装形成纳米颗粒及其响应肿瘤微环境释药的示意图 ${ }^{[73]}$.

Figure 9 (a) Drug release mechanism of prodrug through GSH thiolysis and ROS oxidation ${ }^{[71-73]}$, (b) chemical structure of the ROS-responsive camptothecin prodrugs SN38-s-OEG, SN38-s-IDM and CPT-s-PEG ${ }^{[71-73]}$, (c) schematic illustration of CPT-s-PEG prodrug self-assembly into nanoparticle and its redox-responsive drug release in tumor microenvironment $^{[73]}$. Copyright (2020) American Chemical Society.

OEG: oligo ethylene glycol, IDM: indomethacin

虽然硒醚键具有比硫醚键更快的 ROS 响应机制, 更多的应用在 ROS 响应型 DDS 中 ${ }^{[74-80]}$, 但在前药系统 的报道相对较少 ${ }^{[81-83]} .2018$ 年, Wang 等 ${ }^{[81]}$ 报道了一种含 有硒醚键的 CO 前药, 在 ROS 存在时 Se 发生氧化进而 释放出 $\mathrm{CO}$ 分子(图 10a), 该前药在水溶液中稳定存在, 能够被 $\mathrm{HOCl} 、{ }^{1} \mathrm{O}_{2}$ 和 $\mathrm{O}_{2}^{-}$等活性氧激活, 实验结果表明 该前药可以向 HeLa 和巨噬细胞(RAW264.7)中递送 CO, $\mathrm{CO}$ 前药对心脏/心肌细胞(H9C2)无细胞毒性, 但是与化 疗药物(DOX)联合用药显著增强了 DOX 对 HeLa 细胞的 
毒性. 同年, Sun 课题组 ${ }^{[82]}$ 用硫醚/硒醚键偶联抗癌药物 卡巴他赛(cabazitaxel, CTX)和 OA 制备 CTX 前药(图 $10 \mathrm{~b})$, 进而与 $\mathrm{PPa}$ 光敏剂自组装为纳米粒, 并在外表面 包覆一层 DSPE-PEG $2 \mathrm{k}$ 进行联合治疗(图 10c), 在 ROS 存在或者激光照射下, CTX 能够快速释放并对 4T1 癌细 胞显示出较强的杀伤效果. 值得说明的是, 含硒连接的 前药优于含硫连接的前药分子, 制备的前药纳米系统显 著地延长了 CTX 和 PPa 的循环半衰期和提高了药物在 肿瘤组织的累积量, 从而在活体水平上获得了协同的化 学光动力疗效.

(a)

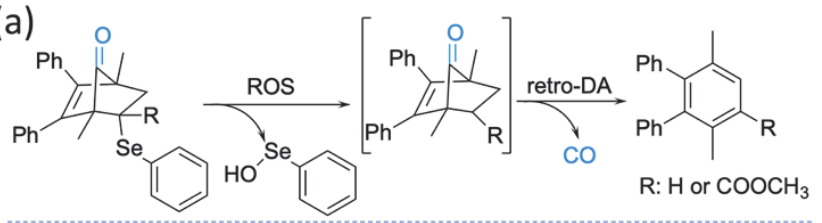

(b)
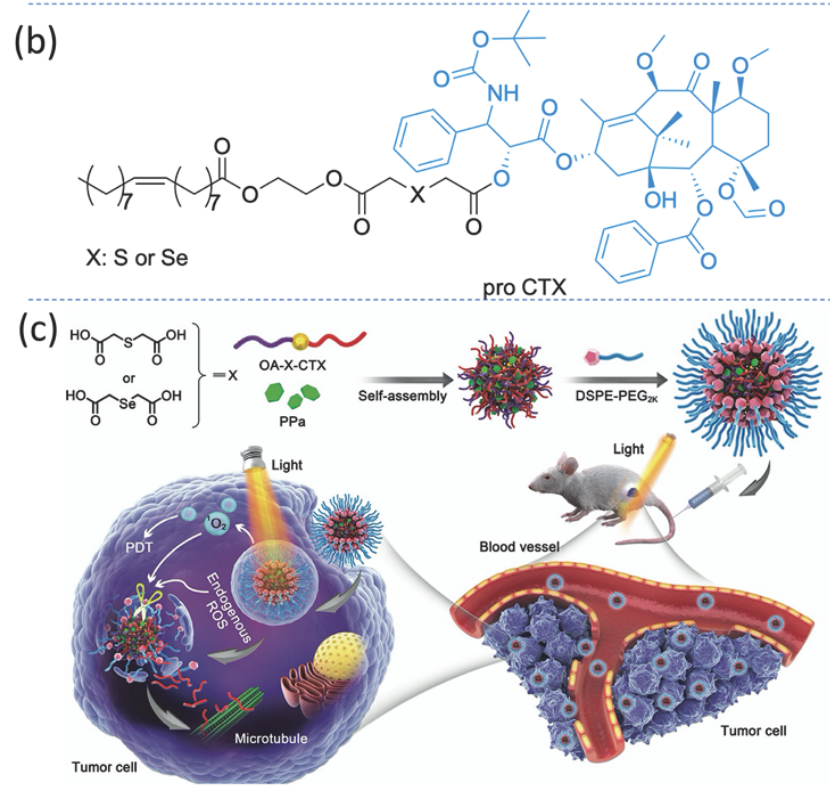

图 10 (a) ROS 响应型 CO 前药化学结构及其 ROS 响应过程 ${ }^{[81]}$, (b) ROS 响应型卡巴他赛前药化学结构 ${ }^{[82]}$, (c) 负载 PPa 的 OA-X-CTX 纳 米粒子自组装及其级联放大释药的示意图 ${ }^{[82]}$.

Figure 10 (a) Chemical structure of the ROS-responsive $\mathrm{CO}$ prodrug and its oxidation process ${ }^{[81]}$, (b) chemical structure of the ROS-responsive cabazitaxel (CTX) prodrugs OA-x-CTX ${ }^{[82]}$, (c) schematic illustration of self-assembly of OA-x-PTX nanoparticle loaded with PPa and its cascade amplification release process ${ }^{[82]}$. Copyright (2018) The Royal Society of Chemistry.

\section{3 硫缩酮基前药}

硫缩酮(TK)因其在酸性和碱性条件下的稳定性而 被广泛用作有机合成中的羰基保护基 ${ }^{[84-86]}$. 但是在氧化 条件下, 硫缩酮可以通过氧化方式被裂解进而释放偶联 的活性分子(图式 3), 这一化学反应激发了研究者探索 其作为 ROS 响应连接臂开发前药的价值 ${ }^{[87-96]}$. Ge 课题 组 ${ }^{[97-99]}$ 在 ROS 响应型前药方面做了很多研究, 2019 年, 他们 ${ }^{[98]}$ 设计合成由 PEG 和含颈基缩酮连接 $\mathrm{CPT}$ 的甲基 丙烯酸单体聚合而成的两亲性嵌段共聚物前药(图 11a), 考虑到肿瘤组织内 ROS 浓度低、分布不均匀等特点, 将 $\beta$-lapa 与前药共组装为纳米颗粒实现肿瘤内源性 ROS 和 NQO1 催化产生的 ROS 共同促进快速释放 CPT 以及 与高 ROS 水平协同抑制肿瘤生长, 实验结果表明该体 系对 $4 \mathrm{~T} 1$ 细胞显示出更低的 $\mathrm{IC}_{50}(0.3 \mu \mathrm{g} / \mathrm{mL}$, 游离的 CPT 为 $0.48 \mu \mathrm{g} / \mathrm{mL}$ ), 在荷瘤小鼠的治疗中, 该体系显示 出优越的生物安全性和高效的肿瘤(4T1)抑制和消融能 力. 同年, 该课题组 ${ }^{[99]}$ 在 CPT-TK-PEG 前药体系中加入 酸敏感的 2 - (五亚甲基) 甲基丙烯酸乙酯 (2-(pentamethyleneimino) ethyl methacrylate, PEMA)形成 新的聚合物前药分子(图 11b), 通过包载超小 $\mathrm{Fe}_{3} \mathrm{O}_{4}$ 纳米 颗粒和葡萄糖氧化酶(glucose oxidase, GOD)组装成多功 能纳米复合体, 该体系在肿瘤特异性微环境中能够快速 消耗葡萄糖生成 $\mathrm{H}_{2} \mathrm{O}_{2}$ 和在酸性环境中溶解 $\mathrm{Fe}_{3} \mathrm{O}_{4}$ 释放 铁离子, $\mathrm{H}_{2} \mathrm{O}_{2}$ 和铁离子之间 Fenton 反应生成了 $\mathrm{HO} \cdot$, 能 够触发前药氧化级联反应并快速释放 CPT 活性药物. 同时，肿瘤酸性环境使得 PEMA 质子化增加纳米粒的膜 透过性(图 11b)，因此，通过葡萄糖等营养物质原位消耗

(a)
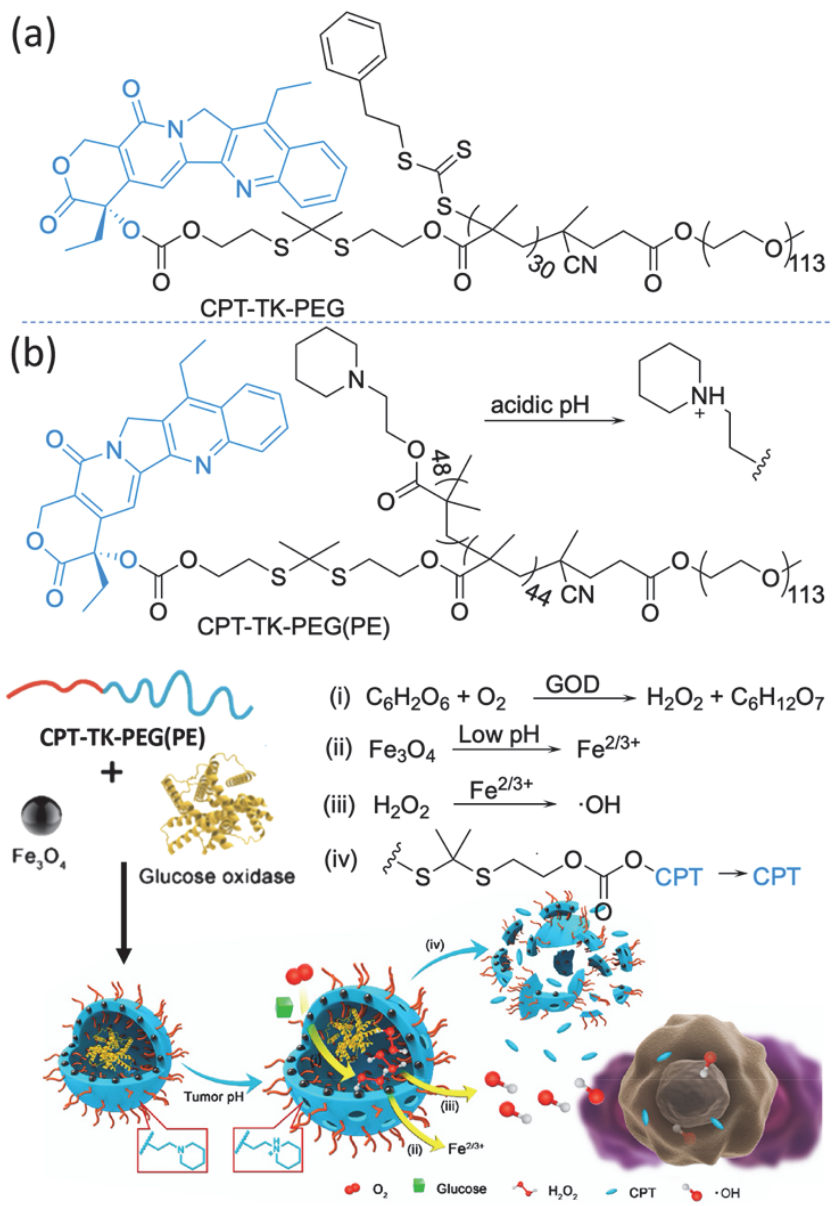

图 11 (a) ROS 响应型喜树碱前药的化学结构 ${ }^{[98]}$, (b) ROS 响应型喜树 碱前药的化学结构、前药自组装为纳米颗粒及其在肿瘤部位响应过 程 ${ }^{[99]}$.

Figure 11 (a) Chemical structure of ROS-responsive CPT prodrug CPT-TK-PEG ${ }^{[98]}$, (b) chemical structure of ROS-responsive prodrug CPT-TK-PEG(PE), self-assembly of prodrugs into nanoparticles and its response function in tumors ${ }^{[99]}$. Copyright (2019) American Chemical Society. 
以及同时生成 ROS, 实现了饥饿疗法、化学动力疗法和 化疗等协同治疗, 以达到高效抑瘤目的, 体外和体内实 验结果确认了该体系比游离 CPT 药物具有更优的治疗 效果. 在 2019 年, Chen 等 ${ }^{[100]}$ 合成由 PEG 和含颈基缩酮 连接抗癌药物 DOX 的甲基丙烯酸单体聚合而成的两亲 性嵌段共聚物前药 (图 12a), 与 $\mathrm{pH}$ 敏感的 PEG-PDPA-PDA 聚合物分子共同包载 $\beta$-lapa, 自组装形 成 $\mathrm{pH} / \mathrm{ROS}$ 双响应型纳米前药, 该体系在细胞内酸性环 境中实现 $\mathrm{pH}$ 触发降解并释放 $\beta$-lapa, 胞内产生的 $\mathrm{H}_{2} \mathrm{O}_{2}$ 通过 Fenton 反应进一步转化为有剧毒的 $\mathrm{HO}$ - 实现化学 动力疗法(chemodynamic therapy, CDT), 随后 ROS 诱导 的硫缩酮连接臂断裂释放 DOX, 体内荷瘤小鼠抗 A549 肿瘤治疗结果表明, ROS 的产生和抗肿瘤药物的释放级 联反应能有效抑制肿瘤生长.

在 ROS 响应型前药设计中, 将能够供给 ROS 的光 敏剂引入硫缩酮前药系统是一种常规策略, 在光照条件 下, 光敏剂产生的 ROS 一部分氧化硫缩酮键释放活性 药物, 另一部分用于光动力治疗 ${ }^{[101-104]}$. 2018 年, Wang 等 ${ }^{[105]}$ 通过硫缩酮键偶联 cRGD 靶向肽修饰的 PEG, 合 成了 PEG 基的 DOX 大分子前药(图 12a), 并用于包载光 敏剂血卟啉(hematoporphyrin, HP)制备肿瘤靶向性的多 功能前药纳米粒(图 12b), 在体外/内激光照射下均成功 诱导 ROS 生成, ROS 进而触发硫缩酮断裂释放出抗癌药 物 DOX, 在口腔鳞状细胞癌(OTSCC)细胞中, 激光照射 后该体系显示出显著的细胞生长抑制和调亡诱导的效 果, 在荷瘤(OTSCC)小鼠中, 纳米颗粒具有良好的肿瘤 靶向性, 通过多种机制在局部激光照射后显著抑制肿瘤 生 长. 同年, Bai 等 ${ }^{[106]}$ 利用硫缩酮键连接亲水性光敏剂 IR700DX 与大麻素药物 mbc94 开发了肿瘤靶向光动力 前药(图 13a), 在 $690 \mathrm{~nm}$ 的光照射下, 光敏剂产生 ROS 一方面进行光动力治疗, 另一方面氧化硫缩酮连接臂释 放 mbc94 药物, 该前药能够靶向 2 型大麻素受体 (cannabinoid receptor type2, $\mathrm{CB}_{2} \mathrm{R}$ )过度表达癌细胞, 与 不可降解大麻素-光敏剂结构相比, 在小鼠脑肿瘤细胞 $\left(\mathrm{CB}_{2}\right.$-mid DBT $)$ 中显示出显著的治疗效果, 而对不表达 $\mathrm{CB}_{2} \mathrm{R}$ 的健康人胚胎肾细胞(HEK-293)几乎无细胞毒 性. 早在 2016 年, Zhang 等 ${ }^{[107]}$ 开发了一种结构更简单的 ROS 响应型多功能光动力前药, 它是由 GEM 与苂光光 敏剂四苯基卟啉(meso-tetraphenylporphyrin, TPP)通过硫 缩酮键直接连接而成, 并用于癌症的影像导向及原位肿 瘤光动力治疗(图 13b), 在红光(658 nm))照射下, TPP 可 产生单线态氧并通过光动力疗法损伤肿瘤细胞，同时硫 缩酮键可被单线态氧裂解导致吉西他滨的级联释放, 通 过化疗造成持续的细胞损伤, 实验结果表明, 与 ROS 不 可断裂的前药相比, 在体外光照 $\left(658 \mathrm{~nm}, 280 \mathrm{~mW} / \mathrm{cm}^{2}\right)$ 下, 该前药作用于 HeLa 癌细胞的细胞调亡和坏死率 (73.8\%)显著提高(对照组仅为 $41.8 \%$ ), 在 H22 小鼠模型 中, 在两种药剂(前药单体和纳米颗粒)给药下均能有效
抑制肿瘤生长，且效果明显优于吉西他滨和 PDT 对照 组，没有明显的系统毒性.

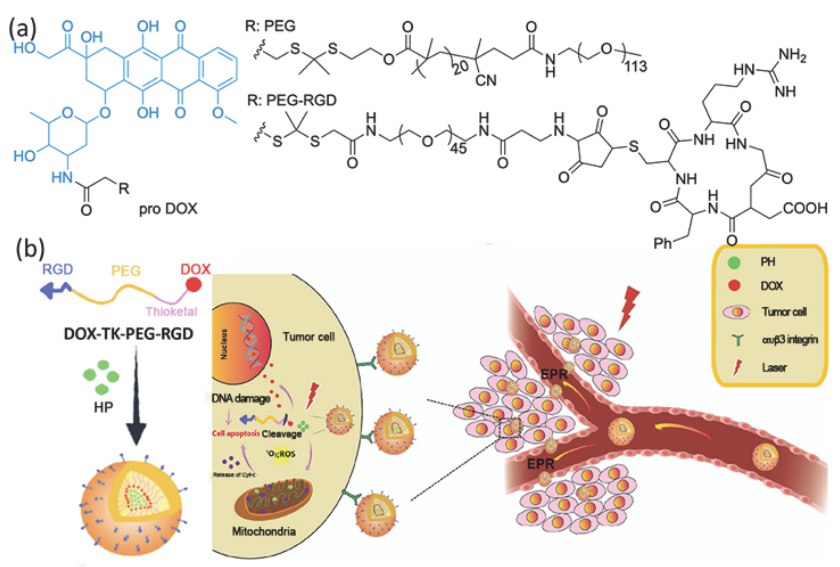

图 12 (a) ROS 响应型阿霉素前药的化学结构 ${ }^{[100,105]}$, (b) 聚合物前药 自组装形成纳米颗粒及靶向抗肿瘤治疗示意图 ${ }^{[105]}$.

Figure 12 (a) Chemical structure of ROS-responsive doxorubicin prodrugs DOX-TK-PEG and DOX-TK-PEG-RGD ${ }^{[100,105]}$, (b) schematic illustration of polymer prodrug self-assembly to form nanoparticles and targeted antitumor therapy ${ }^{[105]}$. Copyright (2018) American Chemical Society.

RGD: cyclo (Arg-Gly-Asp-d-Phe-Cys), HP: hematoporphyrin

(a)
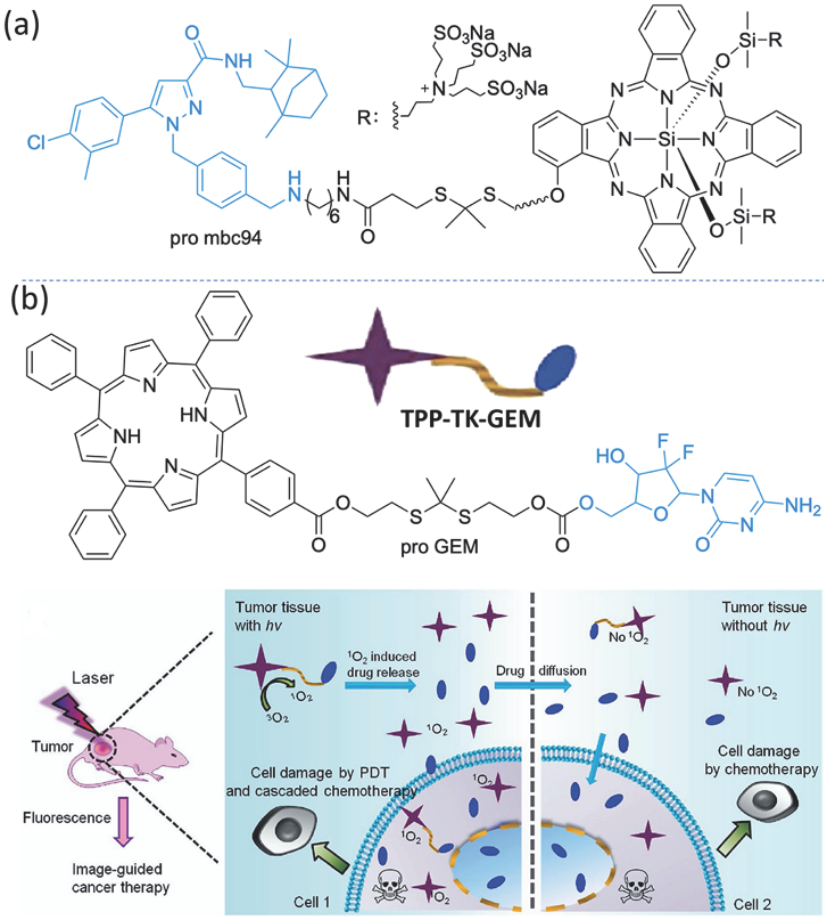

图 13 (a) ROS 响应型光敏剂——大麻素前药的化学结构 ${ }^{[106]}$, (b) ROS 响应型光敏剂——吉西他滨前药的化学结构, 前药用于光动力疗法强 化肿瘤治疗及肿瘤组织化疗的示意图 ${ }^{[107]}$.

Figure 13 (a) Chemical structure of ROS responsive cannabinoid prodrug mbc94-TK-IRD ${ }^{[106]}$, (b) chemical structure of ROS responsive gemcitabine prodrug TPP-TK-GEM, and processes of enhanced tumor therapy by image-guided photodynamic therapy and subsequent chemotherapy in tumors ${ }^{[177]}$. Copyright (2016) WILEY-VCH Verlag GmbH \& Co. $\mathrm{KGaA}$

IRD: IRDyeTM 700DX, TPP: meso-tetraphenylporphyrin, GEM: gemcitabine 


\section{4 过氧草酸酯基前药}

过氧草酸酯容易与氧化剂 $\mathrm{H}_{2} \mathrm{O}_{2}$ 反应生成 1,2-二氧 乙二酮(1,2-dioxetanedione)并迅速分解为二氧化碳, 因 此在 ROS 响应型前药中巧妙地利用过氧乙酸酯键可以 实现 ROS 诱导其降解并释放出活性分子(图式 3) ${ }^{[108-110]}$. 2019 年, Lee 等 ${ }^{[111]}$ 以姜黄素(curcumin, CUR)、草酰氯和 1,4-环己烷二甲醇为原料经一锅缩合反应合成了共聚物 前药(图 14a)并采用单乳液技术制备纳米颗粒, 实验结 果发现该纳米颗粒能够有效清除 $\mathrm{H}_{2} \mathrm{O}_{2}$, 并以 $\mathrm{H}_{2} \mathrm{O}_{2}$ 触发 的方式水解不稳定的草酸酯键并释放姜黄素, 同时产生 的二氧化碳气泡, 增强中毒肝脏的超声对比度, 在活化 细胞中具有良好的抗氧化和抗炎活性. 在此之前, 2017 年, Höcherl 等 ${ }^{[112]}$ 就报道了以抗癌化疗激素类似物己烯 雌酚(diethylstilbestrol, DEB)为原料, 采用一锅法合成了 一种新型活性氧敏感可生物降解聚合物前药(图 14a), 由该前药制备的纳米颗粒可在富含活性氧的环境(例如 癌细胞) 中进行氧化降解释放化疗药物, 实验结果表明 该前药体系能够在 $1 \mathrm{mmol} / \mathrm{L} \mathrm{H}_{2} \mathrm{O}_{2}$ 存在时观察到 DEB 的 快速释放, 体外细胞实验显示该体系对 MCF-7 癌细胞 有显著的细胞毒性, 而对人成纤维细胞(HF)毒性较低. 2019 年, Guan 等 ${ }^{[113]}$ 将鬼臼毒素(podophyllotoxin, POD) 与聚乙二醇丙烯酸单酯通过 $\mathrm{H}_{2} \mathrm{O}_{2}$ 响应草酸酯键连接设 计了一种新型 POD 前药, 并与 DSPE-mPEG 一起自组装 成稳定的纳米颗粒(图 15), 体外实验表明, $\mathrm{H}_{2} \mathrm{O}_{2}$ 能激活 前药纳米粒并使其释放 POD, 对结肠癌(CT26)细胞具 有与原药类似的细胞毒性, 但是对正常细胞(NIH 3T3) 几乎没有细胞毒性(细胞存活率 $>90 \%$ ), 体内荷瘤 (CT26)小鼠研究表明, PEG 化的前药纳米粒能够有效延 长血液循环时间, 可显著提高对肿瘤的治疗效果, 且无 明显的全身毒性.

(a)

(b)
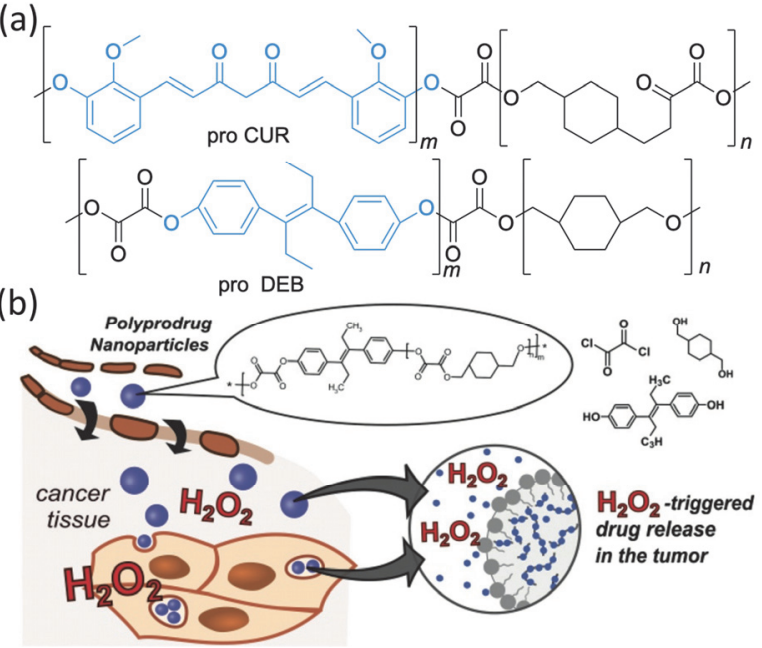

图 14 (a) ROS 响应型聚合物前药的化学结构 ${ }^{[11,112]}$, 药纳米粒在富 $\mathrm{H}_{2} \mathrm{O}_{2}$ 肿瘤微环境中降解和释放药物 ${ }^{[112]}$

(b) 聚合物前

Figure 14 (a) Chemical structure of ROS-responsive polymer prodrugs $^{[111,112]}$, (b) polymer prodrug nanoparticles with self-catalytic degradable polymer and drug release in $\mathrm{H}_{2} \mathrm{O}_{2}$-rich tumor microenvironments ${ }^{[112]}$. Copyright (2017) The Royal Society of Chemistry. CUR: curcumin, DEB: diethylstilbestrol
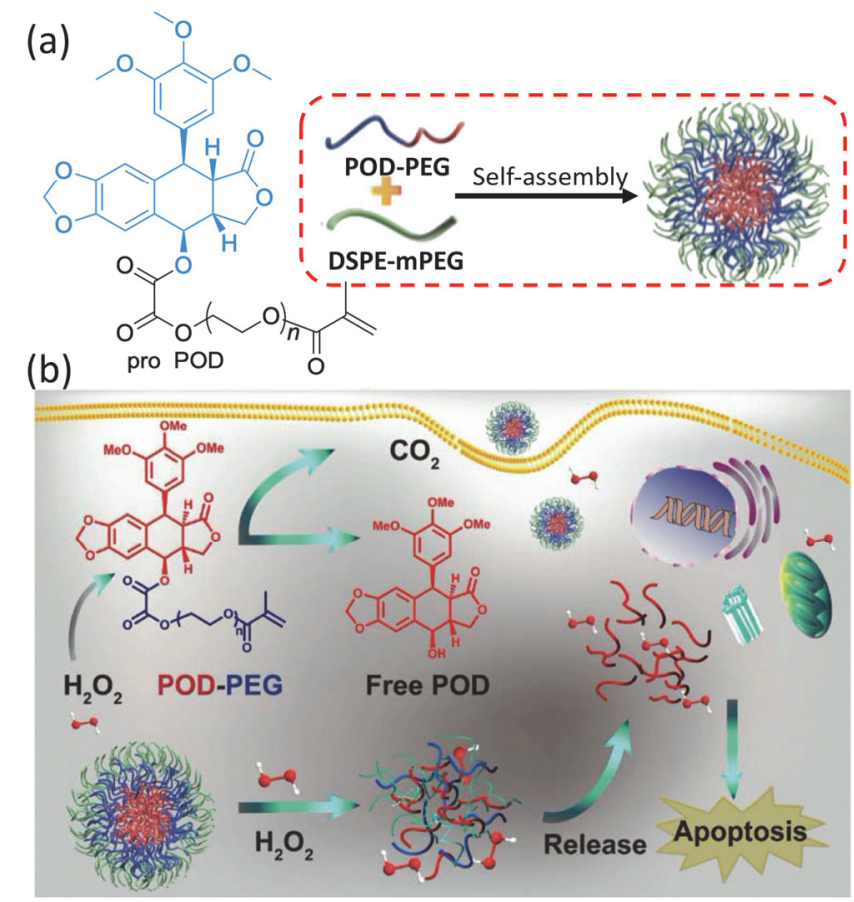

图 15 (a) ROS 响应型鬼臼毒素前药的化学结构及其自组装形成前药 胶束, (b) 肿瘤细胞内 ROS 响应降解和药物释放 ${ }^{[113]}$.

Figure 15 (a) Chemical structure of ROS responsive podophyllotoxin prodrug and its self-assembly into prodrug micelles, (b) ROS responsive degradation and drug release in tumor cells ${ }^{[113]}$. Copyright (2019) The Royal Society of Chemistry.

POD: podophyllotoxin

尽管癌细胞的 $\mathrm{H}_{2} \mathrm{O}_{2}$ 浓度高于正常细胞, 但内源性 $\mathrm{H}_{2} \mathrm{O}_{2}$ 有时候对前药系统仍不足以产生有效的氧化反应, 因此在 $\mathrm{ROS}$ 前药体系中加入活性氧促进剂( $\beta$-lapa、 GOD、光敏剂和抗环血酸)来提供额外的 ROS 的补偿策 略被研究者们所重视 ${ }^{[99,107,114-116]} .2016$ 年, Ge 课题组 ${ }^{[115]}$ 利用草酸酯键将 CPT 偶联到聚乙二醇-聚丙烯酸侧链上 形成 CPT 聚合物前药(图 16a), 并利用棕㭣酰抗坏血酸 酯 ( $L$-ascorbic acid 6-palmitate, PA) 作为肿瘤组织中过氧 化氢生成的促氧化剂制备了 $\mathrm{H}_{2} \mathrm{O}_{2}$ 响应的 CPT 聚合物前 药胶束, 维生素 $\mathrm{C}$ 及其衍生物(如 PA)通常在生理浓度下 充当抗氧化剂, 而在肿瘤组织中的药理学浓度下为促氧 化作用，其中抗坏血酸基团被金属蛋白催化剂氧化为抗 坏血酸自由基, 同时给氧提供一个电子形成超氧自由 基, 最后转化为 $\mathrm{H}_{2} \mathrm{O}_{2}$ (图 16b), 产生的 $\mathrm{H}_{2} \mathrm{O}_{2}$ 一方面通过 氧化应激诱导肿瘤细胞淍亡, 另一方面与内源性 $\mathrm{H}_{2} \mathrm{O}_{2}$ 协同触发 CPT 释放, 体内荷瘤(4T1)小鼠实验结果表明 该体系能够通过氧化和化疗协同作用有效地抑制了肿 瘤生长. 2019 年, Yang 等 ${ }^{[16]}$ 用草酸酯键将 CPT 接枝到 PEG-P(AA-DA)聚合物侧链形成 $\mathrm{pH} / \mathrm{ROS}$ 级联反应型聚 合物前药(图 17a), 并包载 $\beta$-lapa 制备聚合物前药胶束, 构建了一种具有电荷反转能力和自扩增释药模式的纳 米给药系统(图 17b), 在肿瘤弱酸性微环境中, 胶束体系 的表面电荷由负向正转变增强肿瘤细胞的摄取，该胶束 

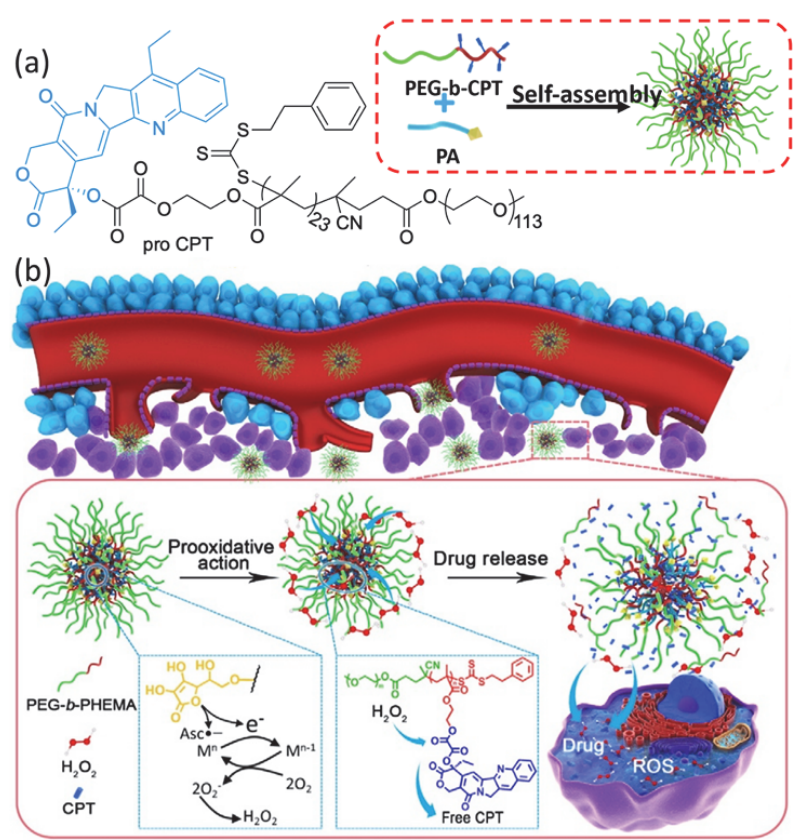

图 16 (a) ROS 响应型喜树碱前药的化学结构及其自组装形成前药胶 束, (b) 前药胶束用于上调肿瘤组织中的过氧化氢水平并触发随后的 CPT 释放以实施协同氧化化疗的示意图 ${ }^{[15]}$.

Figure 16 (a) Chemical structure of ROS-responsive CPT prodrug and its self-assembly into nanoscaled drug delivery micelles, (b) schematic diagram to illustrate the utility of prodrug-based micelles in up-regulating $\mathrm{H}_{2} \mathrm{O}_{2}$ level in tumors and stimulating subsequent CPT release to exert synergistic oxidation-chemotherapy ${ }^{[115]}$. Copyright (2018) Elsevier. PA: palmitoyl ascorbate

(a)

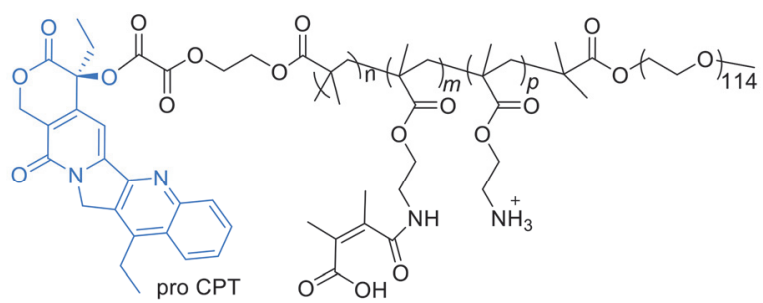

(b)

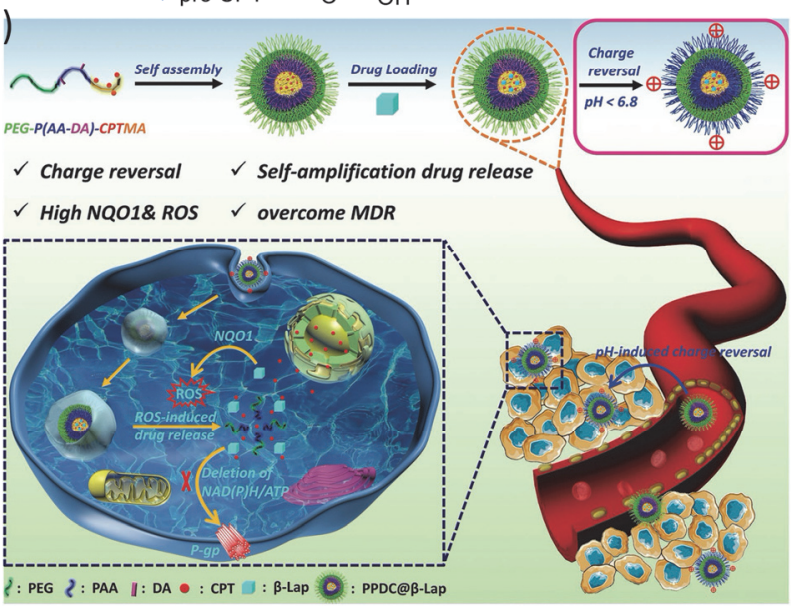

图 17 (a) ROS 响应型喜树碱前药的化学结构, (b) 前药纳米颗粒在体 内肿瘤治疗中的应用 ${ }^{[16]}$.

Figure 17 (a) Chemical structure of ROS-responsive CPT prodrug, (b) illustration of the prodrug nanoparticle with self-amplifiable drug release for tumor therapy in vivo ${ }^{[116]}$. Copyright (2019) WILEY-VCH Verlag $\mathrm{GmbH} \& \mathrm{Co}$. KGaA.

PAA: poly (2-aminoethyl methacrylate hydrochloride), DA: dimethylmaleic anhydride, $\beta$-Lap: $\beta$-lapachone
可以在富含 $\mathrm{ROS}$ 的胞内环境中解离, 释放 $\beta$-lapa 和 $\mathrm{CPT}$, 释放的 $\beta$-lapa 能在 NQO1 的催化下产生活性氧, 诱导胶束的自扩增分解和药物释放，同时产生的过量 ROS 可协同 CPT, 进一步促进肿瘤细胞调亡, 克服肿瘤 细胞的多药耐药, 体内外研究一致表明, $\mathrm{pH}$ 响应性电荷 逆转、肿瘤 ROS 水平上调、ROS 反应性药物释放自增 强的联合作用通过协同氧化化疗达到了有效的抗肿瘤 (MCF-7)效果.

\section{5 其他 ROS 敏感键前药}

氨基丙烯酸酯(AA)中的富电子烯烃能与 ${ }^{1} \mathrm{O}_{2}$ 进行 $[2+2]$ 环加成得到二氧乙烷中间体，二氧乙烷中间体自 发分解释放药物分子(图式 3). 在 2012 年, You 课题 组 ${ }^{[117-126]}$ 开发了一种易合成的氨基丙烯酸酯基连接臂, 基于 $\mathrm{AA}$ 键设计和制备了一系列前药分子用于抗肿瘤治 疗的研究, 并将 AA 基团直接连接光敏剂和抗癌药物形 成的光激活前药的药物释放机制称为 “光解化学” (photo-unclick chemistry). 在一系列前药中, You 等 ${ }^{[124]}$ 合 成了氨基丙烯酸酯连接臂偶联的光敏剂卟啉 (core-modified porphyrin, CMP)和微管蛋白聚合抑制剂 (combretastatin a-4, CA4)级联双激活的光动力前药 CA4-AA-CMP(图 18a), 在红光 $\left(\lambda_{\max }=690 \mathrm{~nm}, 10 \mathrm{~min}\right.$ )照

(a)

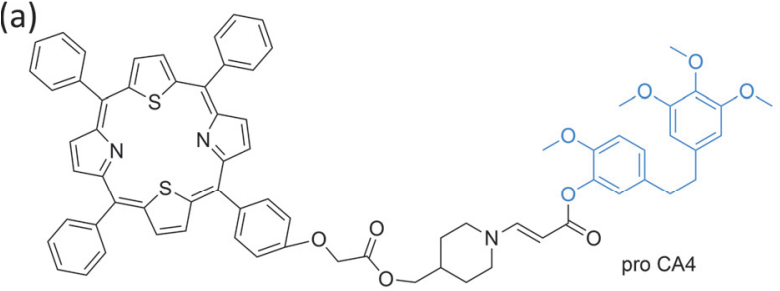

(b)

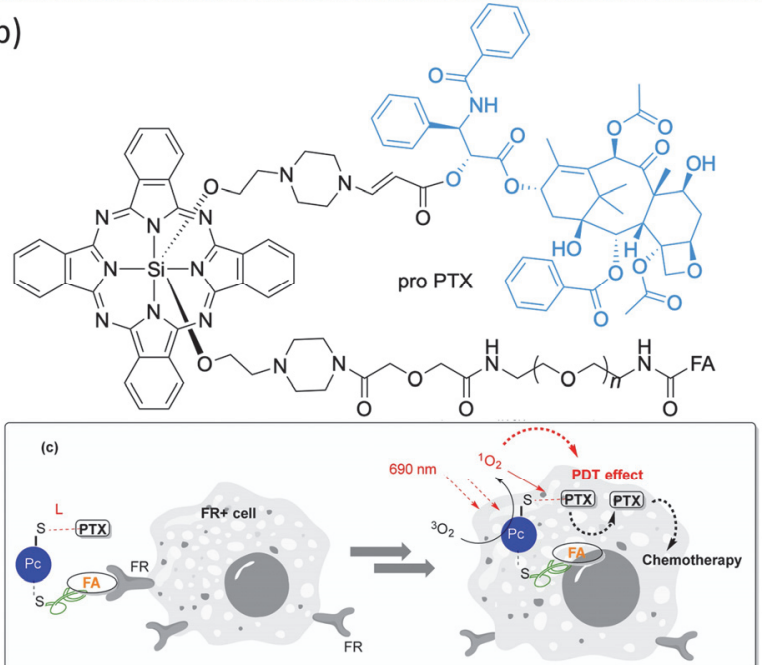

图 18 (a) ROS 响应型抗肿瘤前药的化学结构 ${ }^{[124]}$, (b) ROS 响应型紫 杉醇前药的化学结构及其用于叶酸受体介导的细胞摄取 ${ }^{[126]}$.

Figure 18 (a) Chemical structure of ROS-responsive anti-tumor prodrug of CA4-AA-CMP ${ }^{[124]}$, (b) chemical structure of ROS-responsive PTX prodrug of PTX-AA-PC-FA and folate receptor-mediated cellular uptake and biological consequences upon illumination at $690 \mathrm{~nm}^{[126]}$. Copyright (2017) American Chemical Society.

CA4: combretastatin A-4, CMP: core-modified porphyrin, PC: silicon phthalocyanine, FA: folic acid 
射下该前药有显著的药物释放 $(>80 \%)$, 与未光照实验 组相比, 该前药对 MCF-7 细胞的毒性提升了 6 倍, 在小 鼠移植瘤(Colon 26)模型中显示出明显的体内肿瘤抑制 作用. 在他们的第二代氨基丙烯酸酯基光动力前药中, You 等 ${ }^{[25,126]}$ 通过将光敏剂替换为具有光动力和荧光成 像能力的硅酞菁( silicon phthalocyanine, PC), 成功地与 光学成像结合起来, 在 $\mathrm{PC}$ 分子上通过氨基丙烯酸酯连 接紫杉醇药物和聚乙二醇叶酸(PEG-FA)合成靶向的光 动力前药(图 18b), PEG-FA 的引入提高了药物的溶解度 并增加了肿瘤靶向性, 在叶酸受体表达阳性的 SKOV-3 细胞中观察到前药的有效细胞摄取和更强的光毒性活 性, 体内光学成像证实了前药在肿瘤组织的有效积聚.

利用噻唑烷酮(TZ)对抗癌药物的羧酸进行掩蔽是 另一种开发 ROS 激活前药的方法. 在活性氧存在时, TZ 基团在亲和基团攻击下水解促使噻唑烷酮基团分离, 生 成游离羧基的活性药物分子(图式 3). 2015 年, Cohen 等 ${ }^{[127]}$ 用 $\mathrm{TZ}$ 基团分别屏蔽了布洛芬(ibuprofen, IBU)和一 种 MMPi 的羧基合成了 2 种前药(图 19a), 在 $100 \mathrm{mmol} / \mathrm{L}$ 的 Tris- $\mathrm{HCl}$ 缓冲液中具有良好的稳定性, 并能够响应 $\mathrm{H}_{2} \mathrm{O}_{2}$ 快速释放药物分子, 实验结果表明前药形式对其 靶点几乎没有活性, 但在 $\mathrm{H}_{2} \mathrm{O}_{2}$ 存在下, 其活性几乎全部 恢复. 2018 年, Clausen 等 ${ }^{[128]}$ 合成了一种新型的 $\mathrm{H}_{2} \mathrm{O}_{2}$ 敏 感的 TZ 基 MTX 前药(图 19b), 该前药具有良好的理化 和药代动力学特性, 并能够在病理生理 $\mathrm{H}_{2} \mathrm{O}_{2}$ 浓度下被 激活释放出 MTX 药物分子, 在小鼠 II 型胶原诱导关节 炎(CIA) 模型中显示出与 MTX 相当的治疗效果, 与 MTX 组相比, 治疗组小鼠在研究结束时体重下降较少, 显示出毒性降低的迹象.

(a)
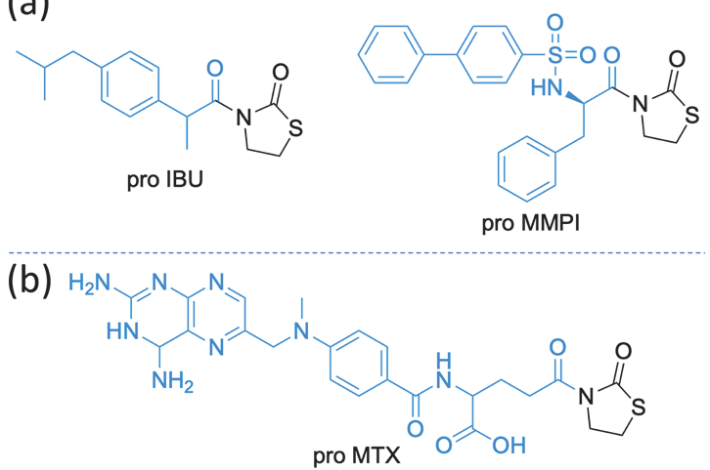

图 19 (a) ROS 响应型布洛芬和基质金属蛋白酶抑制剂前药的化学结 构 ${ }^{[127]}$, (b) ROS 响应型甲氨蝶呤前药的化学结构 ${ }^{[128]}$

Figure 19 (a) Chemical structure of ROS-responsive ibuprofen (IBU) and matrix metalloproteinase inhibitor (MMPi) prodrugs ${ }^{[127]}$, and (b) chemical structure of ROS responsive methotrexate (MTX) prodrug ${ }^{[128]}$

2019 年, Yin 等 ${ }^{[129]}$ 首次报道一种基于 $\alpha$-酮酰胺基的 前药设计和应用(图 20a), 前药中 $\alpha$-酮酰胺部分起着吸 电子基团的作用, 从而屏蔽了氮芥的活性, 在 $\mathrm{H}_{2} \mathrm{O}_{2}$ 存在 时, $\mathrm{H}_{2} \mathrm{O}_{2}$ 亲核攻击酮基随后发生 Baeyer-Villiger 重排, 最终水解释放氮芥药物分子恢复其 DNA 烷基化活性(图 20b). 作者设计并合成了一组基于 $\alpha$-酮酰胺基的氮芥前 药, 其中 KAM-2 前药具有较强的肿瘤细胞生长抑制活 性和对癌细胞的高选择性, 实验验证了 KAM-2 的 $\mathrm{H}_{2} \mathrm{O}_{2}$ 诱导分解作用, 释放的氮芥具有 DNA 损伤和促进细胞 调亡活性. 该工作揭示 $\alpha$-酮酰胺基可作为一种新的前药 敏感基团，并有可能激发未来 ROS 前药的发展.

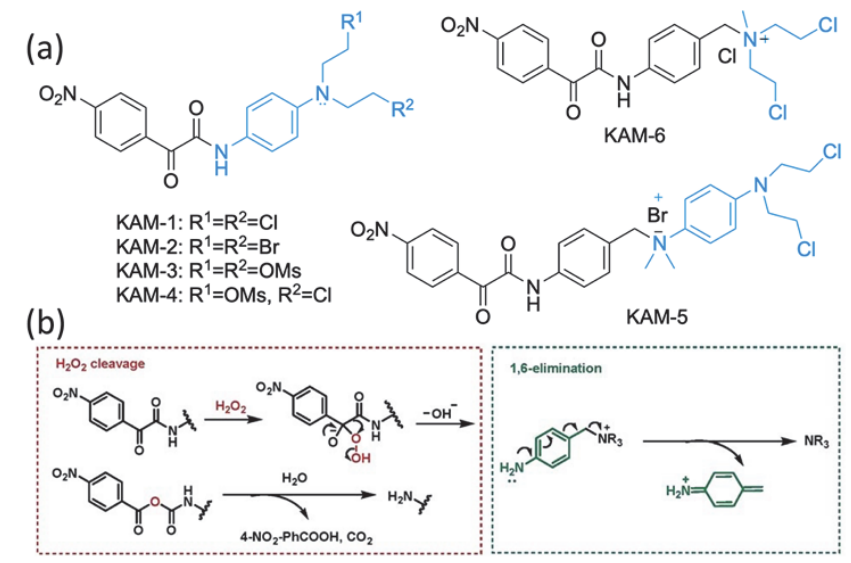

图 20 (a) ROS 响应型 $\alpha$-酮酰胺基氮芥类前药化学结构, (b) $\mathrm{H}_{2} \mathrm{O}_{2}$ 响应 活化生成 DNA 烷基化剂的机理 ${ }^{[129]}$.

Figure 20 (a) Chemical structure of ROS-responsive $\alpha$-ketoamide nitrogen mustard prodrugs, (b) molecular mechanisms of $\mathrm{H}_{2} \mathrm{O}_{2}$-responsive activation to generate DNA alkylating agents ${ }^{[129]}$. Copyright (2019) The Royal Society of Chemistry.

\section{4 结论和展望}

相较于正常组织, 肿瘤微环境中具有独特的组织结 构和代谢特征, 例如高活性氧水平、高 GSH 浓度、低 $\mathrm{pH}$ 值和高表达特定的酶. 基于肿瘤特殊的微环境, 将 抗癌药物与特殊敏感键结合形成的前药，能够抑制或屏 蔽其在肿瘤组织以外的细胞毒性，从而在被肿瘤细胞摄 取后，前药中敏感键的切除以恢复其抗癌活性. 因此, 前药在降低毒副作用和提高肿瘤靶向性治疗等方面显 示出巨大的优势. 近年来, 前药在一系列生物医学应用 (肿瘤或炎症相关疾病的靶向治疗)中取得了十分重要的 阶段性成果，而且在其临床开发和批准方面正在取得惊 人的进展.

自 2010 年第一例 ROS 响应型前药被报道以来，关 于 ROS 响应型前药的研究和报道逐年激增, 伴随着活 性氧敏感键研究的多样化, 越来越多的前药系统被设计 和应用于复杂的生物研究. 在此, 我们综述了基于不同 敏感键设计的 ROS 响应型前药体系在肿瘤治疗方面的 成果，以及引入 $\operatorname{ROS}$ 促进剂在前药递送系统中的应用. ROS 响应型前药的成功开发主要取决于 ROS 敏感键的 敏感性和有效性，不同敏感键的氧化响应机制、响应灵 敏度和在前药中的位置对药物释放动力学有很大的影 响. 就目前报道的 ROS 响应型前药而言, 无论是小分子 前药、载体类前药, 还是引入光敏剂等 ROS 促进剂的多 功能前药, 在体外细胞水平和小鼠肿瘤模型中都显示出 
优于原药的肿瘤特异性杀伤活性和更低的毒副作用. 这 激励着研究者们全身心地投入到优化现有前药和开发 新前药的工作中去, 并最终在临床肿瘤治疗中获得令人 满意的效果.

尽管在肿瘤治疗的 ROS 前药设计和应用方面已经 取得了重大进展，并且在许多方面表现出巨大的优势， 但其真正走进临床以及实际应用还要面临许多障碍和 挑战: (1)相比于原药, 虽然前药的活性减弱, 但是仍然 有一定程度的背景活性. 因为用于构建前药的 ROS 敏 感键化学反应活性高, 难免会存在一定水平的背景水 解, 要实现体内肿瘤特异性激活还需要进一步优化 ROS 敏感键和构建的前药体系. (2) ROS 的病理生理浓 度是否足以确保有效地激活前药. 肿瘤组织处活性氧水 平一般是由较为稳定的 $\mathrm{H}_{2} \mathrm{O}_{2}$ 的浓度来衡量, 其他种类 ROS 浓度变化波动频繁, 因此有时候肿瘤微环境的 ROS 浓度并不能快速地激活前药释放. (3)体内血液循环 和肝代谢等机体调节机制使得其无法获得高效的药物 利用率. 在体内血液循环中, 小分子原药、小分子前药 以及纳米前药, 其中的一部分将会被机体调节机制代谢 清除. 因此, 如何设计前药体系能够使更多的药物累积 在病灶部位并发挥最大的疗效是未来研究者们努力的 方向. 然而, 前药策略的引入极大地提高了药物本身的 效用价值, 而且更多的研究者们在关注和设法解决临床 肿瘤治疗所面临的共同问题，比如引入药物递送系统、 靶向基团、影像导航以及 $\operatorname{ROS}$ 促进剂 $(\beta$-拉帕醌、葡萄 糖氧化酶、光敏剂和抗环血酸等)等. 总之, 尽管存在巨 大的挑战, ROS 响应型前药的设计策略在提高肿瘤特异 性治疗效果和降低全身毒副作用方面展示了极大的优 越性, 并且它将越来越受到生物医学领域研究者的关注 和欢迎。

\section{作者简介}

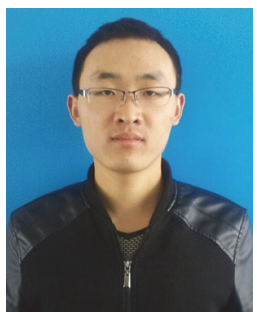

张留伟, 男, 大连理工大学生物化工系在读博士生, 目前 研究方向为生物纳米材料和抗肿瘤前药的设计、制备及其在 肿瘤治疗方面的应用.

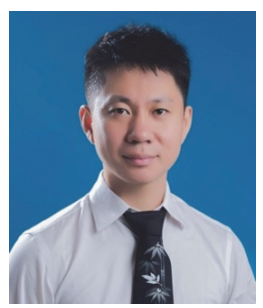

陈鹿先, 男, 大连理工大学生物工程学院特聘研究员, 师
从美国工程院院士、日本东京大学 Prof. Kazunori Kataoka(片 冈一则教授), 曾担任美国麻省理工学院(MIT)化学系 (Prof. Jeremiah A. Johnson 研究室)博士后研究员, 致力于化学、生 物、医学交叉学科研究工作, 目前研究方向包括纳米医学、高 分子自组装、基因治疗等，尤其强调基因/药物递送技术在顽 固性癌症以及中枢神经疾病治疗上的应用价值.

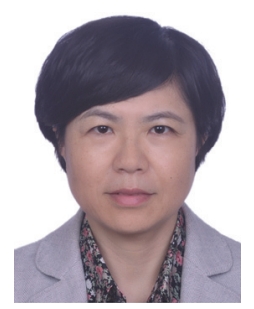

王静云，女，教授，博士生导师. 2001 年毕业于大连理工 大学获得博士学位, 2002 2004 年美国西北大学(Northwestern University)化学系博士后, 主要从事活细胞内生物分子可视化 荧光探针和生物纳米新材料的研究.

\section{References}

[1] Nathan, C.; Ding, A. Cell 2010, 140, 951.

[2] D'Autreaux, B.; Toledano, M. B. Nat. Rev. Mol. Cell Biol. 2007, 8, 813.

[3] Trachootham, D.; Alexandre, J.; Huang, P. Nat. Rev. Drug Discov. 2009, 8,579 .

[4] Schumacker, P. T. Cancer Cell 2015, 27, 156.

[5] Brieger, K.; Schiavone, S.; Miller, F. J., Jr.; Krause, K. H. Swiss Med. Wkly. 2012, 142, w13659.

[6] Costa, A.; Scholer-Dahirel, A.; Mechta-Grigoriou, F. Semin. Cancer Biol. 2014, 25, 23.

[7] Nathan, C.; Cunningham-Bussel, A. Nat. Rev. Immunol. 2013, 13, 349.

[8] Martin, K. R.; Barrett, J. C. Hum. Exp. Toxicol. 2002, 21, 71.

[9] Ahsan, H.; Ali, A.; Ali, R. Clin. Exp. Immunol. 2003, 131, 398.

[10] Andersen, J. K. Nat. Med. 2004, 10, 18.

[11] Haigis, M. C.; Yankner, B. A. Mol. Cell 2010, 40, 333.

[12] Kumar, S. V.; Saritha, G.; Fareedullah, M. Ann. Biol. Res. 2010, 1, 158.

[13] Paravicini, T. M.; Touyz, R. M. Cardiovasc. Res. 2006, 71, 247.

[14] Szatrowski, T. P.; Nathan, C. F. Cancer Res. 1991, 51, 794.

[15] Wellen, K. E.; Hotamisligil, G. S. J. Clin. Invest. 2005, 115, 1111.

[16] Boveris, A.; Alvarez, S.; Bustamante, J.; Valdez, L. Methods Enzymol. 2002, 349, 280.

[17] Giorgio, M.; Trinei, M.; Migliaccio, E.; Pelicci, P. G. Nat. Rev. Mol. Cell Biol. 2007, 8, 722 .

[18] Mueller, S. Biol. Med. 2000, 29, 410.

[19] Stone, J. R.; Yang, S. Antioxid Redox Sign. 2006, 8, 243.

[20] Du, Z.; Zhang, Y.; Ye, J.; Xu, H.; Lang, M. Acta Chim. Sinica 2015, 73, 349. (杜征臻, 张琰，叶金海，徐衡，郎美东，化学学报, 2015, 73, 349.)

[21] Wu, C.; Xie, J.; Quan, J.; Zhu, L. Acta Chim. Sinica 2011, 69, 843. (吴承尧, 谢建刚, 权静, 朱利民, 化学学报, 2011, 69, 843.)

[22] Hou, J.; Li, K.; Qin, C.; Yu, X. Chin. J. Org. Chem. 2018, 38, 612 (后际挺，李坤，覃彩芹，余孝其，有机化学，2018，38，612.)

[23] Jiao, C.; Liu, Y.; Lu, W.; Zhang, P.; Wang, Y. Chin. J. Org. Chem. 2019，39，591. (矫春鹏, 刘媛媛，路文娟，张平平，王延风，有机 化学, 2019, 39, 591.)

[24] Major Jourden, J. L.; Cohen, S. M. Angew. Chem. Int. Ed. 2010, 49 , 6795.

[25] Kuang, Y.; Balakrishnan, K.; Gandhi, V.; Peng, X. J. Am. Chem. Soc. 2011, 133, 19278 .

[26] Chen, W.; Fan, H.; Balakrishnan, K.; Wang, Y.; Sun, H.; Fan, Y.; Gandhi, V.; Arnold, L. A.; Peng, X. J. Med. Chem. 2018, 61, 9132.

[27] Chen, W.; Balakrishnan, K.; Kuang, Y.; Han, Y.; Fu, M.; Gandhi, V.; Peng, X. J. Med. Chem. 2014, 57, 4498.

[28] Cao, S.; Wang, Y.; Peng, X. Chemistry 2012, 18, 3850.

[29] Wang, Y.; Fan, H.; Balakrishnan, K.; Lin, Z.; Cao, S.; Chen, W.; Fan, 
Y.; Guthrie, Q. A.; Sun, H.; Teske, K. A.; Gandhi, V.; Arnold, L. A.; Peng, X. Eur J. Med. Chem. 2017, 133, 197.

[30] Chen, W.; Han, Y.; Peng, X. Chemistry 2014, 20, 7410.

[31] Cao, S.; Wang, Y.; Peng, X. J. Org. Chem. 2014, 79, 501.

[32] Daum, S.; Reshetnikov, M. S. V.; Sisa, M.; Dumych, T.; Lootsik, M. D.; Bilyy, R.; Bila, E.; Janko, C.; Alexiou, C.; Herrmann, M.; Sellner, L.; Mokhir, A. Angew. Chem. Int. Ed. 2017, 56, 15545.

[33] Schikora, M.; Reznikov, A.; Chaykovskaya, L.; Sachinska, O.; Polyakova, L.; Mokhir, A. Bioorg. Med. Chem. Lett. 2015, 25, 3447.

[34] Marzenell, P.; Hagen, H.; Sellner, L.; Zenz, T.; Grinyte, R.; Pavlov, V.; Daum, S.; Mokhir, A. J. Med. Chem. 2013, 56, 6935.

[35] Hagen, H.; Marzenell, P.; Jentzsch, E.; Wenz, F.; Veldwijk, M. R.; Mokhir, A. J. Med. Chem. 2012, 55, 924.

[36] Daum, S.; Chekhun, V. F.; Todor, I. N.; Lukianova, N. Y.; Shvets, Y. V.; Sellner, L.; Putzker, K.; Lewis, J.; Zenz, T.; de Graaf, I. A.; Groothuis, G. M.; Casini, A.; Zozulia, O.; Hampel, F.; Mokhir, A. J. Med. Chem. 2015, 58, 2015.

[37] Daum, S.; Babiy, S.; Konovalova, H.; Hofer, W.; Shtemenko, A.; Shtemenko, N.; Janko, C.; Alexiou, C.; Mokhir, A. J. Inorg. Biochem. 2018, 178, 9

[38] Mu, Y.; Jia, F.; Ai, Z.; Zhang, L. Acta Chim. Sinica 2017, 75, 538. (穆毅, 贾法龙, 艾智慧, 张礼知, 化学学报, 2017, 75, 538.)

[39] Reshetnikov, V.; Daum, S.; Mokhir, A. Chemistry 2017, 23, 5678.

[40] Reshetnikov, V.; Daum, S.; Janko, C.; Karawacka, W.; Tietze, R.; Alexiou, C.; Paryzhak, S.; Dumych, T.; Bilyy, R.; Tripal, P.; Schmid, B.; Palmisano, R.; Mokhir, A. Angew. Chem. Int. Ed. 2018, 57, 11943.

[41] Reshetnikov, V.; Arkhypov, A.; Julakanti, P. R.; Mokhir, A. Dalton Trans. 2018, 47, 6679.

[42] Ai, Y.; Obianom, O. N.; Kuser, M.; Li, Y.; Shu, Y.; Xue, F. ACS Med. Chem. Lett. 2019, 10, 127.

[43] Bhagat, S. D.; Singh, U.; Mishra, R. K.; Srivastava, A. ChemMedChem 2018, 13, 2073.

[44] Biswas, S.; Das, J.; Barman, S.; Rao Pinninti, B.; T, K. M.; Singh, N. D. P. ACS Appl. Mater. Inter. 2017, 9, 28180.

[45] Kumar, R.; Han, J.; Lim, H. J.; Ren, W. X.; Lim, J. Y.; Kim, J. H.; Kim, J. S. J. Am. Chem. Soc. 2014, 136, 17836.

[46] Kim, E. J.; Bhuniya, S.; Lee, H.; Kim, H. M.; Cheong, C.; Maiti, S.; Hong, K. S.; Kim, J. S. J. Am. Chem. Soc. 2014, 136, 13888

[47] Wang, L.; Xie, S.; Ma, L.; Chen, Y.; Lu, W. Eur. J. Med. Chem. 2016, 116,84 .

[48] Liu, H. W.; Hu, X. X.; Li, K.; Liu, Y.; Rong, Q.; Zhu, L.; Yuan, L.; Qu, F. L.; Zhang, X. B.; Tan, W. Chem. Sci. 2017, 8, 7689.

[49] Gao, X.; Cao, J.; Song, Y.; Shu, X.; Liu, J.; Sun, J. Z.; Liu, B.; Tang, B. Z. RSC Adv. 2018, 8, 10975.

[50] Matsushita, K.; Okuda, T.; Mori, S.; Konno, M.; Eguchi, H.; Asai, A.; Koseki, J.; Iwagami, Y.; Yamada, D.; Akita, H.; Asaoka, T.; Noda, T.; Kawamoto, K.; Gotoh, K.; Kobayashi, S.; Kasahara, Y.; Morihiro, K.; Satoh, T.; Doki, Y.; Mori, M.; Ishii, H.; Obika, S. ChemMedChem 2019, 14, 1384.

[51] Peiro Cadahia, J.; Bondebjerg, J.; Hansen, C. A.; Previtali, V.; Hansen, A. E.; Andresen, T. L.; Clausen, M. H. J. Med. Chem. 2018, 61, 3503.

[52] Previtali, V.; Petrovic, K.; Peiro Cadahia, J.; Troelsen, N. S.; Clausen, M. H. Bioorg. Med. Chem. 2020, 28, 115247.

[53] Xu, X.; Liu, K.; Jiao, B.; Luo, K.; Ren, J.; Zhang, G.; Yu, Q.; Gan, Z. J. Control. Release 2020.

[54] Li, M.; Li, S.; Chen, H.; Hu, R.; Liu, L.; Lv, F.; Wang, S. ACS Appl. Mater. Inter. 2016, $8,42$.

[55] Pei, Y.; Li, M.; Hou, Y.; Hu, Y.; Chu, G.; Dai, L.; Li, K.; Xing, Y.; Tao, B.; Yu, Y.; Xue, C.; He, Y.; Luo, Z.; Cai, K. Nanoscale 2018, $10,11418$.

[56] Luan, T.; Cheng, L.; Cheng, J.; Zhang, X.; Cao, Y.; Zhang, X.; Cui, H.; Zhao, G. ACS Appl. Mater. Inter. 2019, 11, 25654.

[57] Lin, M.; Guo, W.; Zhang, Z.; Zhou, Y.; Chen, J.; Wang, T.; Zhong, X.; Lu, Y.; Yang, Q.; Wei, Q.; Han, M.; Xu, D.; Gao, J. Mol. Pharm. 2020, 17, 499

[58] Luo, C. Q.; Zhou, Y. X.; Zhou, T. J.; Xing, L.; Cui, P. F.; Sun, M.; Jin, L.; Lu, N.; Jiang, H. L. J. Control. Release 2018, 274, 56.

[59] Dong, C.; Zhou, Q.; Xiang, J.; Liu, F.; Zhou, Z.; Shen, Y. J. Control. Release 2020, 321, 529.

[60] Gao, F.; Wang, F.; Nie, X.; Zhang, Z.; Chen, G.; Xia, L.; Wang, L. H.; Wang, C. H.; Hao, Z. Y.; Zhang, W. J.; Hong, C. Y.; You, Y. Z. New J. Chem. 2020, 44, 3478.

[61] Wang, M.; Sun, S.; Neufeld, C. I.; Perez-Ramirez, B.; Xu, Q. Angew. Chem. Int. Ed. 2014, 53, 13444.
[62] Li, M.; Zhao, L.; Zhang, T.; Shu, Y.; He, Z.; Ma, Y.; Liu, D.; Wang, Y. Acta Pharm. Sin. B 2019, 9, 421.

[63] Luo, C.; Sun, J.; Liu, D.; Sun, B.; Miao, L.; Musetti, S.; Li, J.; Han, X.; Du, Y.; Li, L.; Huang, L.; He, Z. Nano Lett. 2017, 16, 5401

[64] Sun, B.; Chen, Y.; Yu, H.; Wang, C.; Zhang, X.; Zhao, H.; Chen, Q.; He, Z.; Luo, C.; Sun, J. Acta Biomater. 2019, 92, 219.

[65] Luo, C.; Sun, B.; Wang, C.; Zhang, X.; Chen, Y.; Chen, Q.; Yu, H.; Zhao, H.; Sun, M.; Li, Z.; Zhang, H.; Kan, Q.; Wang, Y.; He, Z.; Sun, J. J. Control. Release 2019, 302, 79.

[66] Wang, K.; Yang, B.; Ye, H.; Zhang, X.; Song, H.; Wang, X.; Li, N.; Wei, L.; Wang, Y.; Zhang, H.; Kan, Q.; He, Z.; Wang, D.; Sun, J. ACS Appl. Mater. Inter. 2019, 11, 18914.

[67] Zhang, D.; Yang, J.; Guan, J.; Yang, B.; Zhang, S.; Sun, M.; Yang, R.; Zhang, T.; Zhang, R.; Kan, Q.; Zhang, H.; He, Z.; Shang, L.; Sun, J. Biomater. Sci. 2018, 6, 2360.

[68] Yang, J.; Lv, Q.; Wei, W.; Yang, Z.; Dong, J.; Zhang, R.; Kan, Q.; He, Z.; Xu, Y. Drug Deliv. 2018, 25, 807.

[69] Yang, B.; Wang, K.; Zhang, D.; Ji, B.; Zhao, D.; Wang, X.; Zhang, H.; Kan, Q.; He, Z.; Sun, J. RSC Adv. 2019, 9, 9260.

[70] Xu, C.; Sun, Y.; Qi, Y.; Yu, Y.; He, Y.; Hu, M.; Hu, Q.; Wu, T.; Zhang, D.; Shang, L.; Deng, H.; Zhang, Z. J. Control. Release 2018, $284,224$.

[71] Wang, J.; Sun, X.; Mao, W.; Sun, W.; Tang, J.; Sui, M.; Shen, Y.; Gu, Z. Adv. Mater. 2013, 25, 3670 .

[72] Sharma, A.; Lee, M. G.; Won, M.; Koo, S.; Arambula, J. F.; Sessler, J. L.; Chi, S. G.; Kim, J. S. J. Am. Chem. Soc. 2019, 141, 15611.

[73] Yin, W.; Ke, W.; Lu, N.; Wang, Y.; Japir, A.; Mohammed, F.; Wang, Y.; Pan, Y.; Ge, Z. Biomacromolecules 2020, 21, 921.

[74] Ma, N.; Li, Y.; Xu, H.; Wang, Z.; Zhang, X. J Am. Chem. Soc. 2010, $132,442$.

[75] Cao, W.; Gu, Y.; Li, T.; Xu, H. Chem. Commun. (Camb) 2015, 51, 7069.

[76] Liu, J.; Pang, Y.; Zhu, Z.; Wang, D.; Li, C.; Huang, W.; Zhu, X.; Yan, D. Biomacromolecules 2013, 14, 1627.

[77] Ma, N.; Li, Y.; Ren, H.; Xu, H.; Li, Z.; Zhang, X. Polym. Chem. 2010, $1,1069$.

[78] Ma, N.; Xu, H.; An, L.; Li, J.; Sun, Z.; Zhang, X. Langmuir 2011, $27,5874$.

[79] Tian, Y.; Zheng, J.; Tang, X.; Ren, Q.; Wang, Y.; Yang, W. Part. Part. Syst. Charact. 2015, 32, 547.

[80] Li, T.; Yi, Y.; Xu, H. Acta Chim. Sinica 2014, 72, 1079. (李天予, 易 宇, 许华平, 化学学报, 2014, 72, 1079.)

[81] Pan, Z.; Zhang, J.; Ji, K.; Chittavong, V.; Ji, X.; Wang, B. Org. Lett. 2018, 20,8.

[82] Yang, B.; Wang, K.; Zhang, D.; Sun, B.; Ji, B.; Wei, L.; Li, Z.; Wang, M.; Zhang, X.; Zhang, H.; Kan, Q.; Luo, C.; Wang, Y.; He, Z.; Sun, J. Biomater. Sci. 2018, 6, 2965.

[83] Li, Y.; Li, Y.; Ji, W.; Lu, Z.; Liu, L.; Shi, Y.; Ma, G.; Zhang, X. J. Am. Chem. Soc. 2018, 140, 4164.

[84] Ganguly, N.; Barik, S. Synthesis 2009, 2009, 1393.

[85] Ling, X.; Zhang, S.; Shao, P.; Wang, P.; Ma, X.; Bai, M. Tetrahedron Lett. 2015, 56, 5242 .

[86] Wilson, D. S.; Dalmasso, G.; Wang, L.; Sitaraman, S. V.; Merlin, D.; Murthy, N. Nat. Mater. 2010, 9, 923.

[87] Xu, C.; Song, R.; Lu, P.; Chen, J.; Zhou, Y.; Shen, G.; Jiang, M.; Zhang, W. Int. J. Nanomed. 2020, 15, 65.

[88] Li, S.; Xie, A.; Li, H.; Zou, X.; Zhang, Q. J. Control. Release 2019, $316,66$.

[89] Xu, L.; Yang, Y.; Zhao, M.; Gao, W.; Zhang, H.; Li, S.; He, B.; Pu, Y. J. Mater. Chem. B 2018, 6, 1076.

[90] Wang, Y.; Zhang, Y.; Ru, Z.; Song, W.; Chen, L.; Ma, H.; Sun, L. J. Nanobiotechnol. 2019, 17, 91

[91] Zhao, Z.; Wang, W.; Li, C.; Zhang, Y.; Yu, T.; Wu, R.; Zhao, J.; Liu, Z.; Liu, J.; Yu, H. Adv. Funct. Mater. 2019, 29, 1909013.

[92] Zhou, F.; Feng, B.; Wang, T.; Wang, D.; Cui, Z.; Wang, S.; Ding, C.; Zhang, Z.; Liu, J.; Yu, H.; Li, Y. Adv. Funct. Mater. 2017, 27, 1703674.

[93] Yuan, Y.; Liu, J.; Liu, B. Angew. Chem. Int. Ed. 2014, 53, 7163.

[94] Xu, X.; Saw, P. E.; Tao, W.; Li, Y.; Ji, X.; Bhasin, S.; Liu, Y.; Ayyash, D.; Rasmussen, J.; Huo, M.; Shi, J.; Farokhzad, O. C. Adv. Mater. 2017, 29, 1700141.

[95] Lamb, B. M.; Barbas, C. F., 3rd Chem. Commun. (Camb) 2015, 51, 3196.

[96] Wang, G.; Zhou, Z.; Zhao, Z.; Li, Q.; Wu, Y.; Yan, S.; Shen, Y.; Huang, P. ACS Nano 2020, 14, 4890.

[97] Ke, W.; Lu, N.; Japir, A.; Zhou, Q.; Xi, L.; Wang, Y.; Dutta, D.; 
Zhou, M.; Pan, Y.; Ge, Z. J. Control. Release 2020, 318, 67.

[98] Yin, W.; Ke, W.; Chen, W.; Xi, L.; Zhou, Q.; Mukerabigwi, J. F.; Ge, Z. Biomaterials 2019, 195, 63 .

[99] Ke, W.; Li, J.; Mohammed, F.; Wang, Y.; Tou, K.; Liu, X.; Wen, P.; Kinoh, H.; Anraku, Y.; Chen, H.; Kataoka, K.; Ge, Z. ACS Nano 2019, 13, 2357.

[100] Wang, S.; Yu, G.; Wang, Z.; Jacobson, O.; Lin, L. S.; Yang, W.; Deng, H.; He, Z.; Liu, Y.; Chen, Z. Y.; Chen, X. Angew. Chem. Int. Ed. 2019, 58,14758

[101] Han, K.; Zhu, J. Y.; Wang, S. B.; Li, Z. H.; Cheng, S. X.; Zhang, X. Z. J. Mater. Chem. B 2015, 3, 8065 .

[102] Pei, P.; Sun, C.; Tao, W.; Li, J.; Yang, X.; Wang, J. Biomaterials 2019, 188,74

[103] Phua, S. Z. F.; Xue, C.; Lim, W. Q.; Yang, G.; Chen, H.; Zhang, Y.; Wijaya, C. F.; Luo, Z.; Zhao, Y. Chem. Mater. 2019, 31, 3349.

[104] Xia, X.; Yang, X.; Huang, P.; Yan, D. ACS Appl. Mater. Inter. 2020, $12,18301$.

[105] Shi, S.; Zhang, L.; Zhu, M.; Wan, G.; Li, C.; Zhang, J.; Wang, Y.; Wang, Y. ACS Appl. Mater. Inter. 2018, 10, 29260.

[106] Ling, X.; Zhang, S.; Liu, Y.; Bai, M. J. Biomed. Opt. 2018, $23,1$.

[107] Liu, L. H.; Qiu, W. X.; Li, B.; Zhang, C.; Sun, L. F.; Wan, S. S.; Rong, L.; Zhang, X. Z. Adv. Funct. Mater. 2016, 26, 6257.

[108] Li, J.; Li, Y.; Wang, Y.; Ke, W.; Chen, W.; Wang, W.; Ge, Z. Nano Lett. 2017, 17, 6983.

[109] Kwon, J.; Kim, J.; Park, S.; Khang, G.; Kang, P. M.; Lee, D. Biomacromolecules 2013, 14, 1618.

[110] Qiao, Z.; Liu, H. Y.; Zha, J. C.; Mao, X. X.; Yin, J. Polym. Chem. 2019, 10, 4305.

[111] Berwin Singh, S. V.; Jung, E.; Noh, J.; Yoo, D.; Kang, C.; Hyeon, H.; Kim, G. W.; Khang, G.; Lee, D. Nanomedicine 2019, 16, 45.

[112] Höcherl, A.; Jäger, E.; Jäger, A.; Hrubý, M.; Konefał, R.; Janoušková, O.; Spěváček, J.; Jiang, Y.; Schmidt, P. W.; Lodge, T. P.; Štěpánek, P. Polym. Chem. 2017, 8, 1999.

[113] Ou, K.; Kang, Y.; Chen, L.; Zhang, X.; Chen, X.; Zheng, Y.; Wu, J.; Guan, S. Biomater. Sci. 2019, 7, 2491.
[114] Wang, S.; Wang, Z.; Yu, G.; Zhou, Z.; Jacobson, O.; Liu, Y.; Ma, Y.; Zhang, F.; Chen, Z.; Chen, X. Adv. Sci. 2019, 6, 1700141.

[115] Li, J.; Ke, W.; Wang, L.; Huang, M.; Yin, W.; Zhang, P.; Chen, Q.; Ge, Z. J. Control. Release 2016, 225, 64.

[116] Dai, L.; Li, X.; Duan, X.; Li, M.; Niu, P.; Xu, H.; Cai, K.; Yang, H. Adv. Sci. 2019, 6, 1801807.

[117] Bio, M.; Nkepang, G.; You, Y. Chem. Commun. (Camb) 2012, 48, 6517.

[118] Hossion, A. M.; Bio, M.; Nkepang, G.; Awuah, S. G.; You, Y. ACS Med. Chem. Lett. 2013, 4, 124.

[119] Bio, M.; Rajaputra, P.; Nkepang, G.; You, Y. J. Med. Chem. 2014, 57,3401 .

[120] Rajaputra, P.; Bio, M.; Nkepang, G.; Thapa, P.; Woo, S.; You, Y. Bioorg. Med. Chem. 2016, 24, 1540.

[121] Thapa, P.; Li, M.; Bio, M.; Rajaputra, P.; Nkepang, G.; Sun, Y.; Woo, S.; You, Y. J. Med. Chem. 2016, 59, 3204.

[122] Bio, M.; Rajaputra, P.; Lim, I.; Thapa, P.; Tienabeso, B.; Hurst, R. E.; You, Y. Chem. Commun. (Camb) 2017, 53, 1884

[123] Li, M.; Thapa, P.; Rajaputra, P.; Bio, M.; Peer, C. J.; Figg, W. D.; You, Y.; Woo, S. J. Pharmacokinet. Pharmacodyn. 2017, 44, 521.

[124] Bio, M.; Rajaputra, P.; Nkepang, G.; Awuah, S. G.; Hossion, A. M.; You, Y. J. Med. Chem. 2013, 56, 3936.

[125] Nkepang, G.; Bio, M.; Rajaputra, P.; Awuah, S. G.; You, Y. Bioconjug. Chem. 2014, 25, 2175.

[126] Thapa, P.; Li, M.; Karki, R.; Bio, M.; Rajaputra, P.; Nkepang, G.; Woo, S.; You, Y. ACS Omega 2017, 2, 6349

[127] Perez, C.; Monserrat, J. P.; Chen, Y.; Cohen, S. M. Chem. Commun. (Camb) 2015, 51, 7116

[128] Andersen, N. S.; Peiro Cadahia, J.; Previtali, V.; Bondebjerg, J.; Hansen, C. A.; Hansen, A. E.; Andresen, T. L.; Clausen, M. H. Eur. J. Med. Chem. 2018, 156, 738 .

[129] Meng, T.; Han, J.; Zhang, P.; Hu, J.; Fu, J.; Yin, J. Chem. Sci. 2019, 10,7156 .

(Cheng, B.) 